\title{
Experimental and Theoretical Research on a Ammonia Pulsating Heat Pipe: new full visualization of flow pattern and operating mechanism study
}

\author{
Zhi Hu Xue*, Wei Qu \\ China Academy of Aerospace Aerodynamics (CAAA), Beijing 100074, China
}

\begin{abstract}
This paper presents a novel study on performance of pulsating heat pipe using ammonia as working fluid. Firstly, a new full visualization experiment with high speed camera is conducted, to study the relationship between the flow patterns and thermo-hydrodynamics of the PHP. The tested PHP, consisting of 6 turns, is fully made of quartz glass tubes with 6 mm outer diameter and $2 \mathrm{~mm}$ inner diameter. The filling ratio for ammonia fluid is $70 \%$. Wall temperature fluctuations of several key positions are recorded under a series charge of heat transfer rates, which are from $25 \mathrm{~W}$ to $520 \mathrm{~W}$. In visualization results, the motion identities, flow pattern variations, breakup and coalescence between the vapor plugs and liquid slugs are illustrated and discussed. In addition, the actual velocity of the vapor is computed from the experimental measurement in this paper, which is never found in other literature but is very important for the mechanism study in theoretical model.

Furthermore, a theoretical model including the dynamical characteristics and heat transfer is investigated coupled with the thermal driving force, friction force and capillary force variations as the flow patterns changed at different transport powers, which are derived from the full visualization experiment. The theoretical results are compared with the experimental results analytically, and the operating mechanism of PHP will be discussed in detail finally.
\end{abstract}

Keywords: Pulsating heat pipe; Ammonia fluid; Heat transfer; Visualization; Flow pattern; Velocity capture; Operating mechanism

\section{INTRODUCTION}

The Pulsating Heat Pipe (PHP), also called Oscillating Heat Pipe (OHP), is considered as one of the promising technologies for a high heat transfer device on small space in electronics cooling due to its high heat transport capability, simple structure without wick, compact size and low cost of manufacturing ${ }^{[1-6]}$. PHP will have a wide engineering application in electronics cooling, furthermore, becoming an ideal candidate for space application via lower weight than conventional heat pipe $\mathrm{e}^{[7-12]}$.

Although simple in its construction, PHP operating mechanism seems very complicated and has been studied in theoretical and experiment by many researchers ${ }^{[13-35]}$. The experimental studies are main focused on the following issues: about the parameter effects of tube diameter, filling ratio, number of turns, inclination angle, and working fluid properties; the flow visualization to observe the oscillating motion, vapor generation, liquid nucleation boiling, coalescence of bubbles, flow direction variation, and phase volume fraction of vapor bubbles and liquid slugs; the thermal performance including temperature fluctuations and thermal resistance. While theoretical investigations attempt to analytically and numerically model the fluid dynamics and/or heat transfer related with oscillating two-phase flow, by means of many simplifying assumptions including one-dimensional models, one flow pattern such as slug flow, a straight tube neglecting the effect of bends, and no effects of surface tension and gravity. These literature are rare cared about different flow patterns corresponding to different working states of PHP and representing the different operating mechanisms, which are needed to consider at the same time in the theoretical model for better understanding the mechanism and main parameter effects of PHP.

The main objective of this paper is to investigate the relationship between the flow patterns and the thermo-hydrodynamics of PHP, thereby determining its operating mechanism at different transport powers, by means of two implements of experimental and theoretical study. Firstly, a new full visualization experiment with high speed camera is conducted, to obtain a series of more elaborate and specific characteristics of flow pattern variations at different transport powers and provide some basic and useful information for theoretical model. At the same time, the actual flowing velocity of the fluids in ammonia PHP that represents hydrodynamics of PHP is tested in the visual experiment, which is never found in other literature, but is very important for the mechanism study in theoretical model. Furthermore, a theoretical model including the dynamical characteristics and heat transfer is proposed, coupled with the thermal driving force, friction force and capillary force variations as the flow patterns variation which are derived from the full visualization experiment. The theoretical results 
are compared with the experimental results analytically to verify its validity, and finally the operating mechanism of PHP is discussed in detail. These investigations may be helpful for us to promote the knowledge of flow and heat transfer features of PHP, and extend our understanding the working mechanisms for explanation some indistinct phenomena or issues in PHP research.

\section{EXPERIMENTAL INVESTIGATION}

\subsection{Experimental Setup}

The schematic of the tested PHP constituting 6 meandering turns is shown in Fig. 1. The prototype is fully made of quartz glass capillary tubes with the total length of $320 \mathrm{~mm}$ from the top to the bottom ${ }^{[35]}$. The inner and outer diameters of the glass tube are 2 $\mathrm{mm}$ and $6 \mathrm{~mm}$, respectively. The radius of each turn is $10 \mathrm{~mm}$. The evaporator section of the PHP is heated by electrical wires which are wrapped on the outer wall surface of the tubes. The total length of heating section is $100 \mathrm{~mm}$. The condenser part of the PHP is cooled by cooling water in cooler box which is circulated by a cold bath appliance. In order to achieve the full visualization of PHP, the upper cover plate of cooler box is also made of quartz glass. The inlet temperature of cooling water is maintained at $17^{\circ} \mathrm{C}$ with $\pm 1^{\circ} \mathrm{C}$ accuracy. The length of cooling section is $100 \mathrm{~mm}$. The motions of vapor bubbles and liquid slugs are photographed and recorded by a high speed CCD camera. Due to the limitation between photo area and high-resolution photos, two photo zones are selected as shown in Fig. 2, including zone I in adiabatic section and zone II in heating section. There are 8 thermocouples located as shown in Fig. 1 to measure the temperature fluctuations of the evaporator, adiabatic and condenser sections of the PHP and the inlet and outlet of cooler box. Four thermocouples T1 to T4 (accuracy $\pm 0.5^{\circ} \mathrm{C}$ ) are located in different tubes to give an average value of evaporator outlet temperature. Two thermocouples T5 and T6 (accuracy $\pm 0.5^{\circ} \mathrm{C}$ ) are placed in the corresponding tubes to provide an average temperature of condenser. The left two thermocouples T7 and T8 (accuracy $\pm 0.2^{\circ} \mathrm{C}$ ) are used to measure the temperature variation of water in inlet and outlet of cooler box. All temperature data are recorded by a highly sensitive temperature logger (Agilent 34970A with resolution $0.1{ }^{\circ} \mathrm{C}$ ) and connected to a PC for scanning the data every one second. Fig. 3 gives the real PHP full visualization platform, which could be adjusted the inclination angle of PHP from $-90^{\circ}$ to $+90^{\circ}$. Fig. 4 shows one real experiment heating test at high input power when the whole heating wires are turned into the red color.

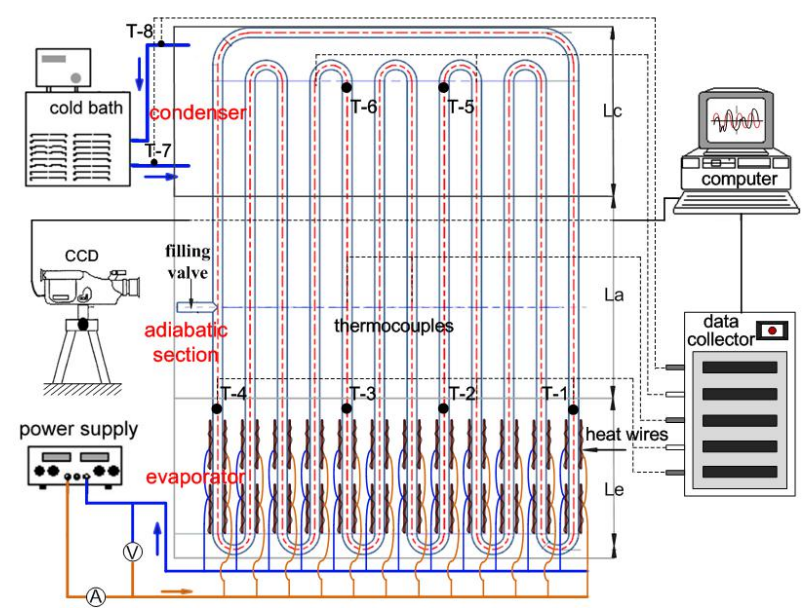

Fig. 1. Schematic of full visualization setup.

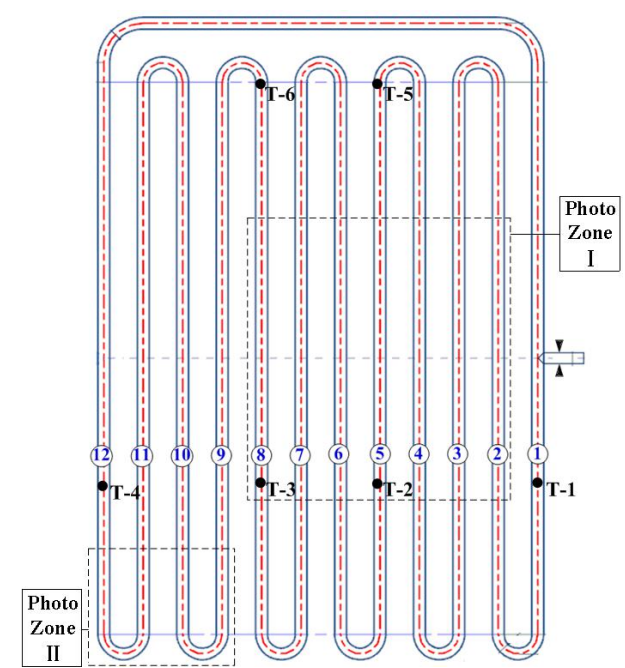

Fig. 2. The diagram of two photo zone.

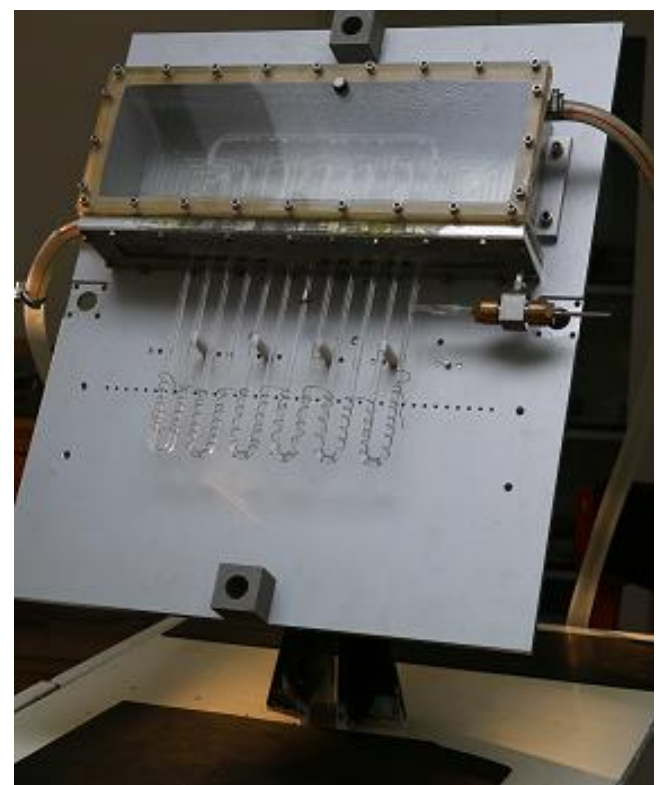

Fig. 3. The real PHP full visualization platform 


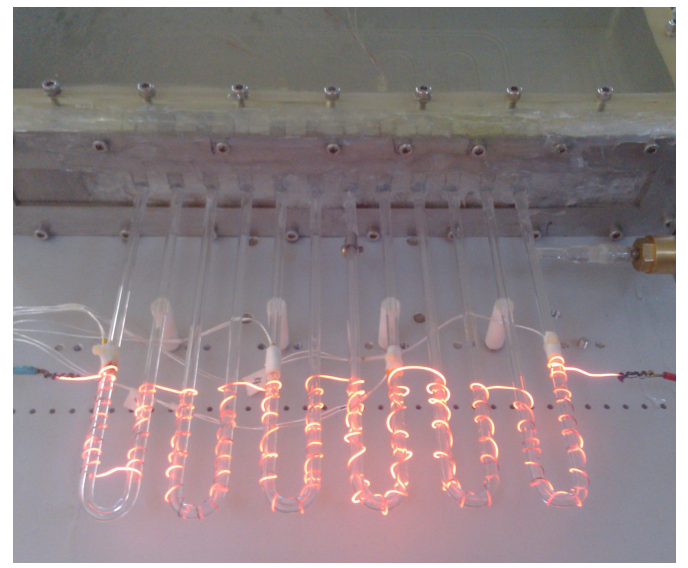

Fig. 4. The real experiment heating test

\subsection{Working Fluid Selection}

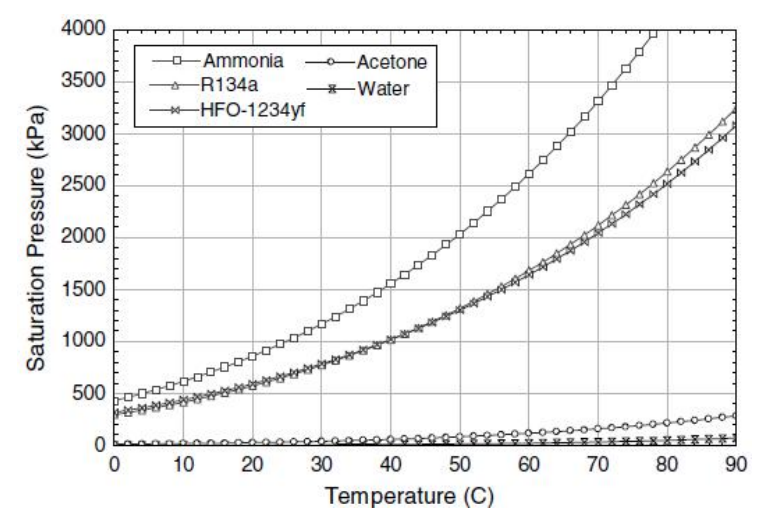

Fig. 5. Saturation pressure as a function of temperature

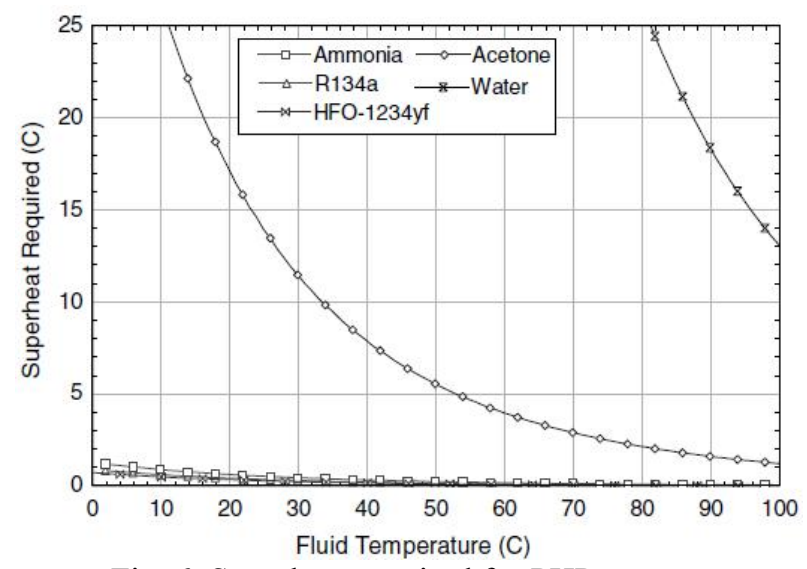

Fig. 6. Superheat required for PHP start-up

Up to date, it is found that the working fluids of PHP employed always are water, ethanol, methanol, acetone, R-123, etc. But for ammonia, there is rarely used. Nevertheless, ammonia has much more advantages than these working fluids ${ }^{[36]}$. It has much higher value of $(\mathrm{d} p / \mathrm{d} T)_{\text {sat }}$, much lower superheat required for start-up, as shown in Fig. 5 and Fig. 6 respectively, little lower dynamic viscosity and surface tension than those fluids. In addition, ammonia PHP is more easier to realize the visualization of flow patterns and its variation range with small input power and low temperature. Therefore, the ammonia is selected as the working fluid in this paper, and could be regarded as one kind of excellent fluids for PHP research and application.

\subsection{Thermal Response Identities}

The heat transport performance of the ammonia PHP with a filling ratio (FR) of $70 \%$ at different inclination angles $\theta$ of $30^{\circ}, 60^{\circ}$ and $90^{\circ}$ are illustrated in Fig. 7 to Fig. 9, respectively. The total power transiting from evaporator to condenser is computed as $25 \mathrm{~W}$ to $520 \mathrm{~W}$ corresponding the increment of heat load. The largest uncertainty of transport power is $\pm 46.7 \mathrm{~W}$ at the state of $520 \mathrm{~W}$.

It could be seen that the temperatures of evaporator and condenser behave fluctuation continually from three figures. This phenomenon could be explained by the local oscillations of vapor bubbles and liquid slugs in capillary, even at the state of circulation for PHP. The ammonia PHP could easily start up even the total transport power is only $25 \mathrm{~W}$, in the case of each capillary tube is contributed with $2.083 \mathrm{~W}$. Of course, it is obvious that the start-up time is decreased as the inclination angle increased by comparing Fig. 9 with Fig. 7. After the start-up, the PHP operating trends including the temperature growth curves, and flow patterns transit are similar, no matter what the inclination angle is, as the increment of input power. Therefore, the thermal performance results at the inclination angle of $60^{\circ}$ will be selected in the following section to assess the flow pattern identities and compare with the theoretical analysis results.

It is noted that the temperature fluctuations and levels for different measurement points in evaporator outlet placements arise obviously different as the transport power increases. And these phenomena will magnify as the inclination angle increases. Because in high power state for PHP, A large one-direction circulation is formed and the flow pattern will be changed remarkably (discussed in detail in the visualization section). When the PHP works in the large one-direction circulation, the flow direction of working fluid in the tubes related with thermocouples $\mathrm{T} 1$ and $\mathrm{T} 2$ is absolutely inverse to that in the tubes related with thermocouples $\mathrm{T} 3$ and $\mathrm{T} 4$. As a result, the temperature level of fluids coming from condenser measured by thermocouples $\mathrm{T} 1$ and $\mathrm{T} 2$ is much 
lower than that coming from evaporator measured by thermocouples T3 and T4 as shown in Fig. 9.

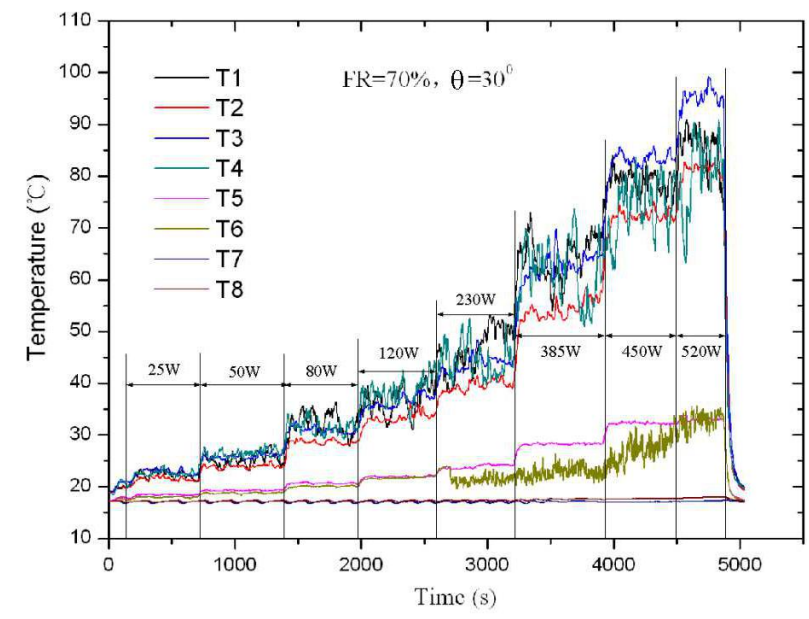

Fig. 7. Temperature response to heat load at inclination angle of $30^{\circ}$

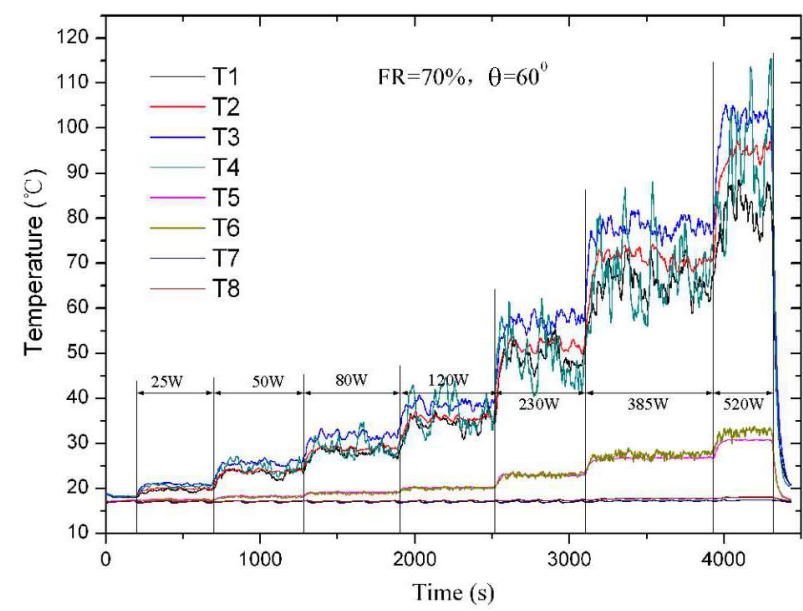

Fig. 8. Temperature response to heat load at inclination angle of $60^{\circ}$

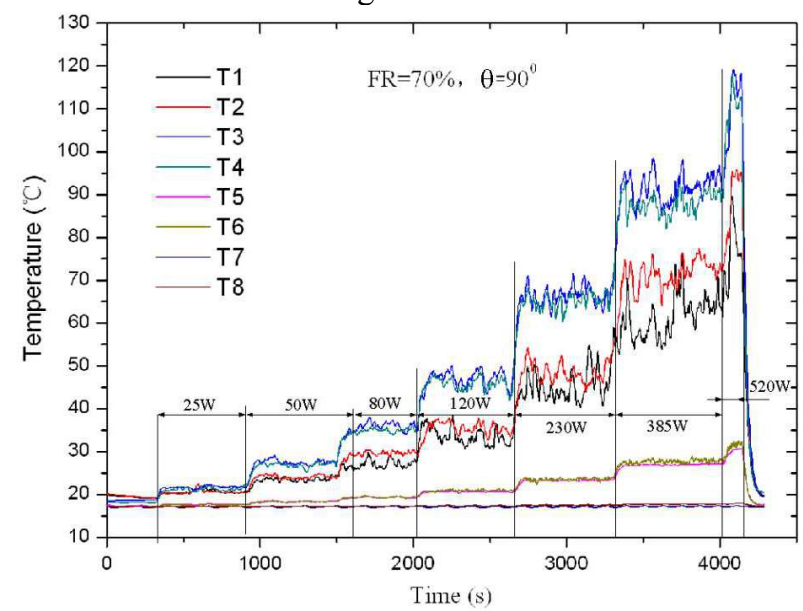

Fig. 9. Temperature response to heat load at inclination angle of $90^{\circ}$

\subsection{Flow Pattern Visualization}

From the full visualization experiment of PHP, it is very easy to observe the start-up characteristics, the local oscillating motions of working fluid, the vapor bubbles generation in heating section, the liquid phase increment after flowing the condenser, the whole distributions of vapor bubbles and liquid slugs in the all capillaries, and the flow direction variations and single one-direction circulation forming for the whole PHP. In the test, it is found that, for PHP operating, its thermo-hydrodynamic characteristics and thermal performance have a direct relationship with the distributions of vapor bubbles and liquid slugs which are called the flow patterns in the tubes. In the other words, at the different working modes corresponding to the variations of the heat transfer rate, the flow patterns of the PHP will be changed to adapt the increment of the transport power, sequentially leading to the variations of temperature fluctuation, thermal resistance of whole PHP, the flow directions of fluid, and the flow velocity of fluid. Therefore, the study on the variation of the flow patterns is significant for us to understand the operating mechanism of PHP, especially for the theoretical or numerical simulations when at the building model of heat transfer and flow equations.

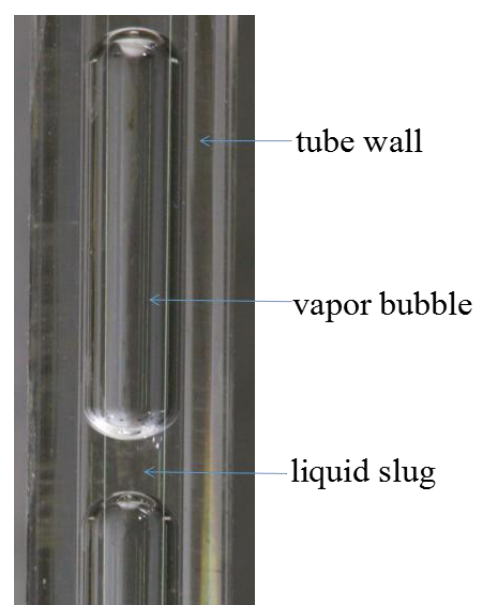

Fig. 10. Static vapor-liquid distribution in zone I

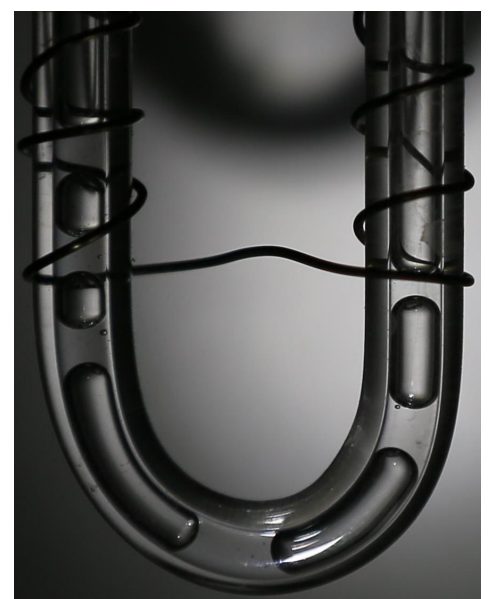

Fig. 11. Static vapor-liquid distribution in zone II 
Fig. 10 and Fig. 11 give the static vapor-liquid distribution in zone I and zone II, respectively. It is seen that in static condition, the vapor bubbles are very regular with two equal spherical surface in end, and the distribution of fluids behaves as one vapor bubble and one liquid slug alternately just like a "train", and the volume fraction of liquid phase is nearly uniform at different tubes of PHP. In two Figures, the interface between the vapor bubble and liquid slug is quite clear, and the static capillary contact angle between them is computed as $65^{\circ}$.

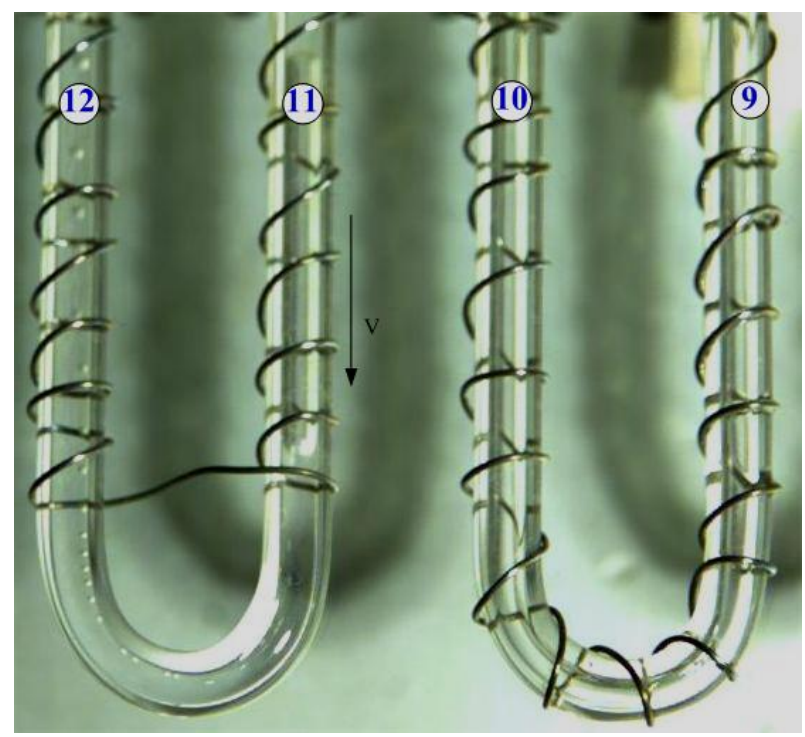

Fig. 12. Visualization identities at low power state of 25 W

Fig. 12 shows the flow pattern and capillary contact angle identities in the tubes numbered 9 to 12 at the transport power of $25 \mathrm{~W}$. At low power state, the capillary contact angle is changed a little, as shown in tube 11 . The advancing contact angle that locates in the head of the vapor is a bit smaller than the static contact angle, while the receding contact angle that locates in the end of the vapor is slightly bigger than the static contact angle. It could be seen that the flow pattern at low power state is not varied compared with the static mode for PHP, with vapor bubbles and liquid slugs alternate distribution and continuous oscillating, and the volume ratio of them without obvious change. This flow pattern is known as the "Slug Flow". At low power state, there are a lot of small scale vapor bubbles generated in the heating section as shown in tube 12 . These small round vapors are floating in a long liquid column, and no coalescence between them, because of low velocity of fluid flowing and vapor generation, and little size of bubbles. This is also the explanation that the whole flow pattern of PHP will not be changed.

As the input power enhances step by step, the oscillating motions of the fluids get more and more violent, and the velocity of the fluids also grows, consequently the shape of vapor bubbles and capillary contact angle will be changed a lot. However, the whole flow pattern is still in the "Slug Flow", not reaching the turning edge of forming stable one-direction circulation. When the heat transfer rate of PHP increases to medium power, for example $80 \mathrm{~W}$, these variations will been the biggest in "Slug Flow" state, and a temporary one-direction circulation will be formed, but not been sustained and quickly restore to oscillating motion.

Fig. 13 and Fig. 14 show visualization identities in zone I and zone II at medium power state of 80 W, respectively. In Fig. 13, the size and number of vapor bubbles, and the volume fraction of vapor phase are all increased at medium transport power in contrast to that at low transport power, as shown in tube 2. And the advancing contact angle of these bubbles becomes sharp towards the flow direction. From the Fig. 13, it can be found that the distribution of vapors and liquids is still like a "train", although each section length of the train is changed a lot; that is to say the flow pattern is still in "Slug Flow". When the PHP operates at medium power state, the condensation of fluids in condenser becomes enhanced, with the volume fraction of liquid phase increasing from the condenser as shown in tube 3 . This phenomenon denotes that the flow pattern in next power step will be changed, and PHP will work towards the high power state. Fig. 14 gives the breakup processes of the long bubbles in the left bend at medium power state. Fig. 14(a) and (b) show that when a long bubble flows through a bend, there will be a concave cavity appeared, generated by the high heat flux and strong inertia force at this area. The concave cavity grows up and the number of cavities increases after the liquid injects along the thin liquid film under the gravity effect, as shown in Fig. 14(c). Eventually, the long bubble will be broken into two or more short bubbles. As seen in the right bend of Fig. 14(c) and (d), the vapor-liquid interface is also different from that at low power state, with the advancing contact angle more smaller, and receding contact angle more larger, resulting to reduce the moving capillary resistance for creating the condition of large onedirection circulation. 


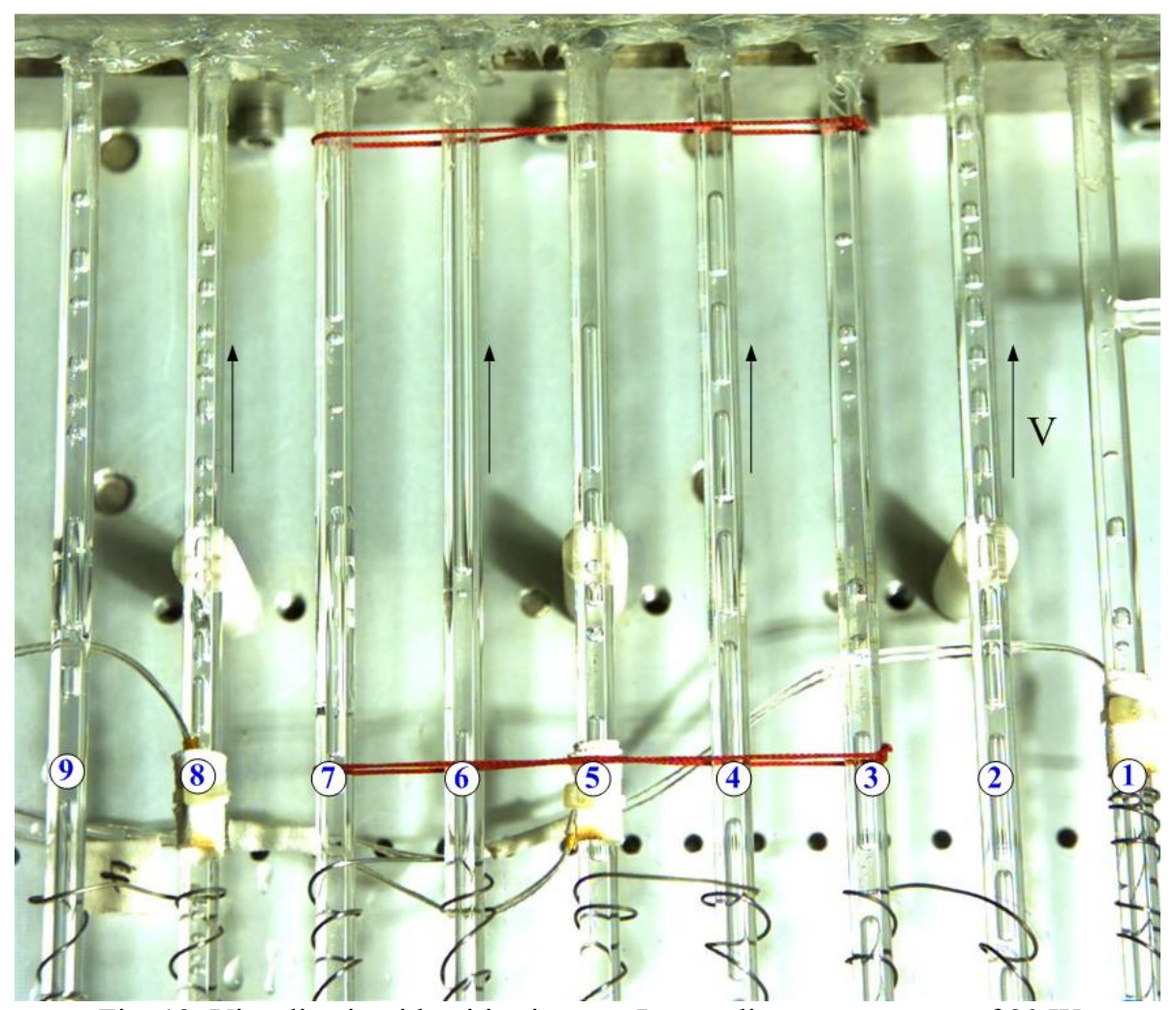

Fig. 13. Visualization identities in zone I at medium power state of $80 \mathrm{~W}$

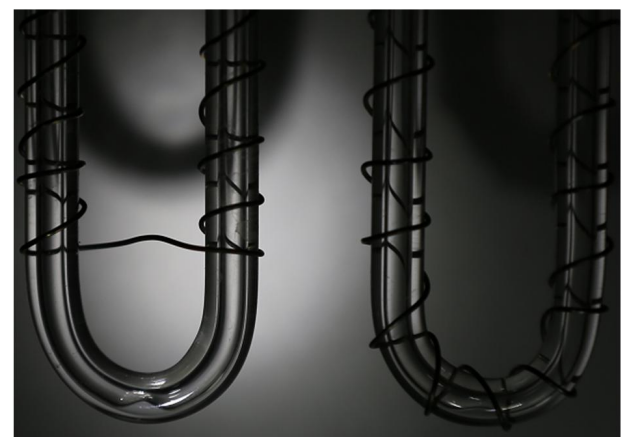

(a)

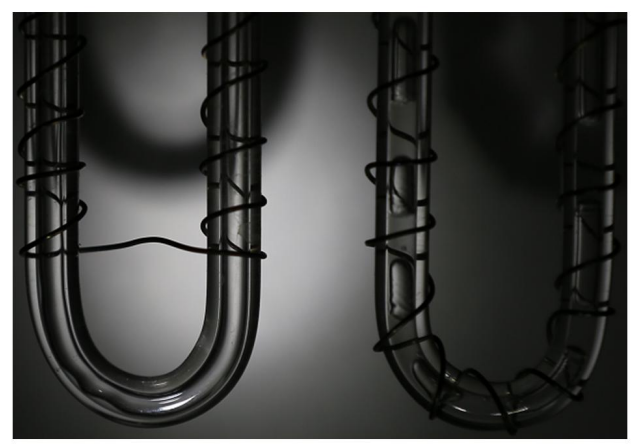

(c)

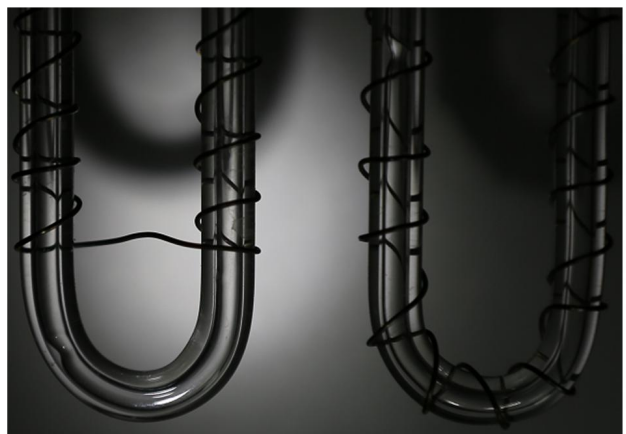

(b)

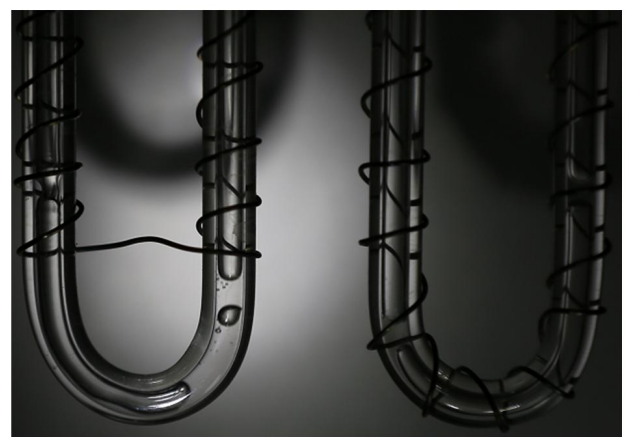

(d)

Fig. 14. Visualization identities in zone II at medium power state of $80 \mathrm{~W}$ 


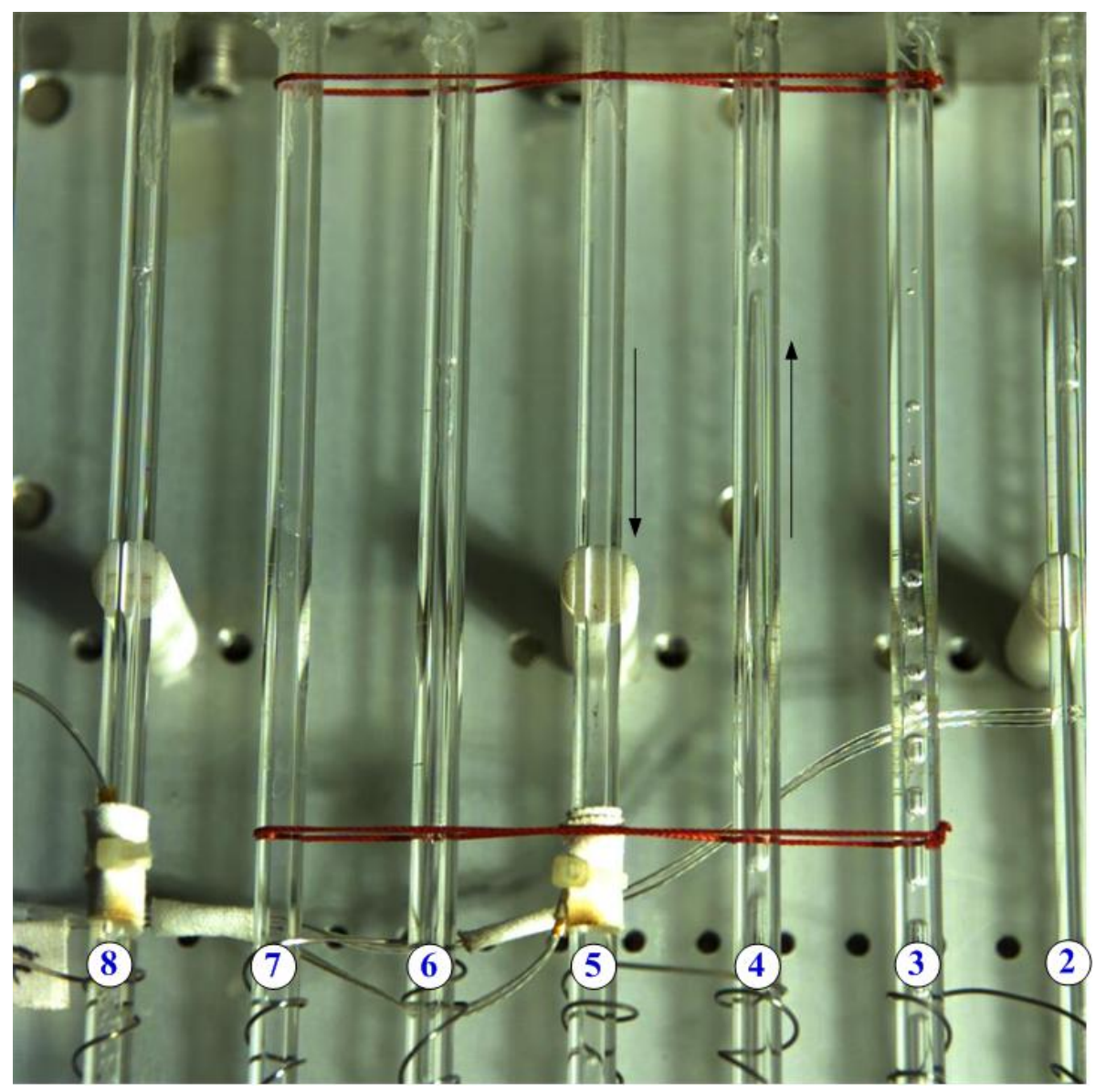

Fig. 15. Visualization identities in zone I at high power state of $520 \mathrm{~W}$

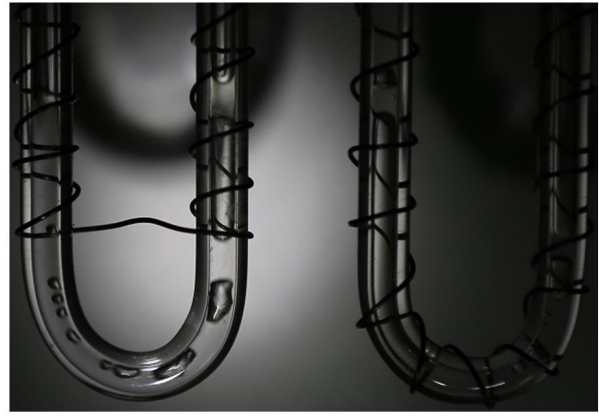

(a)

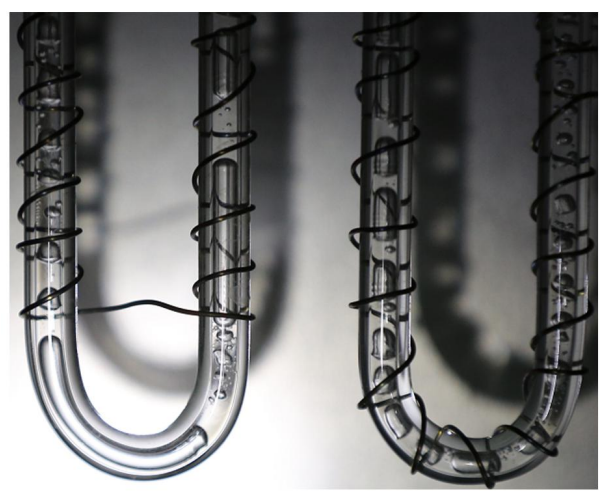

(c)

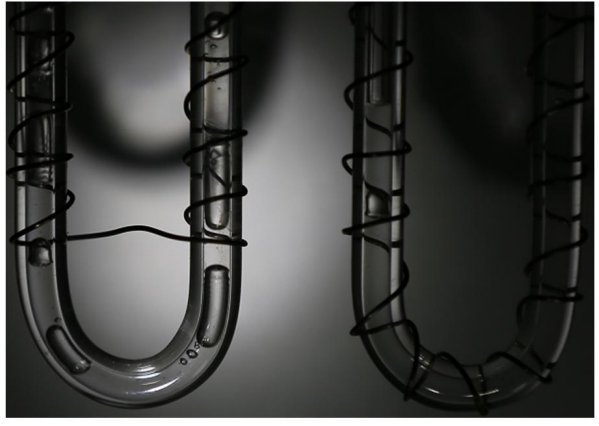

(b)

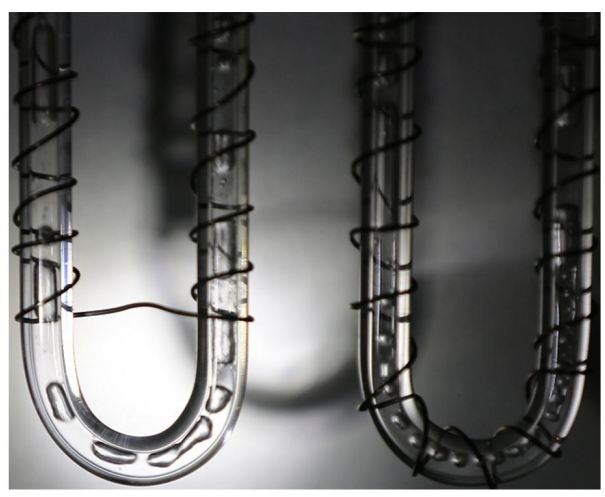

(d)

Fig. 16. Visualization identities in zone II at high power state of $520 \mathrm{~W}$ 


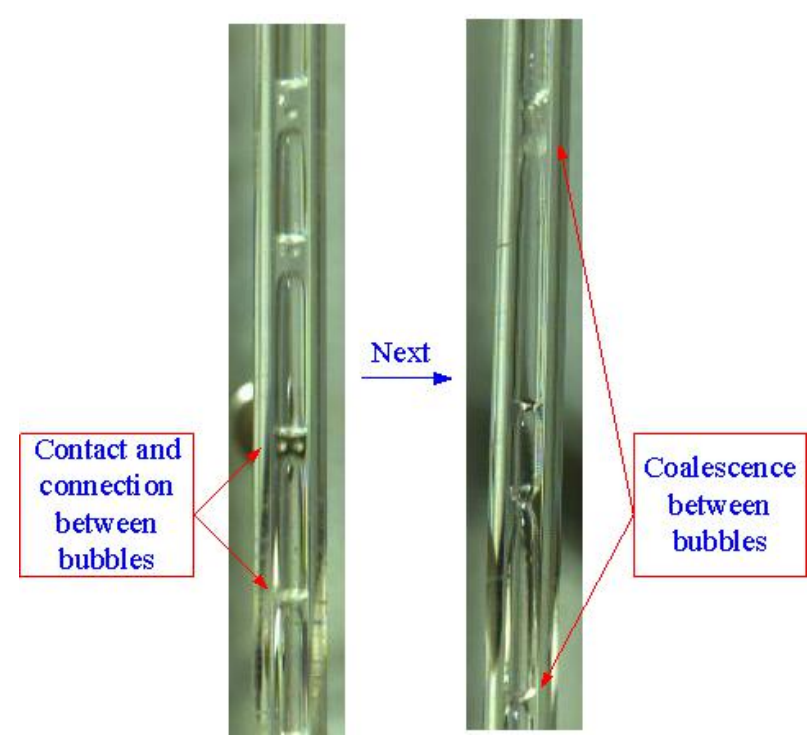

Fig. 17. Connection and coalescence between bubbles in zone I at high input power

If the transport power continuously increases after the medium power mode, the operating state of PHP will be changed significantly. In this case, when the liquid flows through the heating areas, a large number of bubbles will be generated, accumulated and then quickly merged with the surrounding bubbles to form a big bubble, as shown in Fig. 16(c) and (d). The visualization shows that the volume fraction of vapor phase and the generation velocity of vapor bubbles both are dramatically increased, sequentially the saturated vapor pressure or driving force of PHP is quite reinforced. Therefore, a large one-direction circulation appears in whole PHP, and the flow pattern of PHP is varied a lot. Fig. 15 shows the flow pattern variations at the high power state of $520 \mathrm{~W}$. In the tubes where the fluids run from the heating section to cooling section (for example in tube 2, 4, 6 and 8), there are almost the long vapor bubbles occupied the whole tube, while liquid phase left only with the liquid film rounding the vapor. Obviously, after a great many of bubbles leave the evaporator of PHP, the different vapor bubbles will contact, connect the head of one bubble to the end of another bubble, and coalescence finally, as shown in Fig. 17. This vapor-liquid distribution presents a typical flow pattern as "Annular Flow". On the contrary, in the tubes where the fluids come from the condenser to evaporator (such as tube 5 and 7), the whole tube is nearly filled with the pure liquid. In the other words, the vapor bubbles are absolutely condensed after passing through the condenser and turned into liquid phase. It is a perfect loop and one-direction circulation can be sustained, that all the liquid entering into the evaporator will translate into the many vapors rapidly, coalescence between the bubbles, and turn into long vapor bubble after leaving evaporator, and then condensate into the liquid again at condenser. In those processes, some other identities of vapor bubbles are characterized that the long bubbles in adiabatic section behave like a "shuttle" or "bullet", with a very pointed head and a very dull end; and the small bubbles in heating section are irregular shape, floating with the high speed flow in the tube.

\subsection{Fluid Flowing Velocity Capture}

In order to well characterize the different flow patterns and reflect the hydrodynamics of fluids, the velocity of the vapor and liquid is captured in the test. It is the first attempt to measure the velocity of PHP fluids, and seems very interesting and meaningful for later theoretical model verification and the guideline of PHP mechanism.

The velocity computation in physics is derived from the ratio of unit length to unit time. In the test, a selected length $\Delta L$ is used for reference at first. Then a CCD high speed camera with high frame rate is selected to record the motions of vapor bubbles in the zone I or zone II. Thereby, the time $\Delta t$ passing the length $\Delta L$ for a vapor bubble can be computed by dividing the frames and frame rate after experiment.

Thus, the actual velocity of the fluid is,

$$
V=\frac{\Delta L}{\Delta t}
$$

A main operating feature of pulsating heat pipe is the oscillating motion in the tubes no matter what input power is. The direction of oscillating motions will be changed as a spring, and the magnitude of oscillating speed will be different, with a nadir level and a peak level occurred. Fig. 18 gives the nadir and peak velocity of fluids in zone II at low power state. The heat wires in tube 11 is selected as the reference with $\Delta L=45 \mathrm{~mm}$. From the visualization experiment, it is seen that when the fluids in tube 11 flow upwards, the velocity is small. The time $\Delta t$ passing through the length $\Delta L$ for a bubble head as denoted by the red circle in Fig. 18(a) is obtained of $0.36 \mathrm{~s}$. In the test, the accuracy of $\Delta L$ and $\Delta t$ are $\pm 0.5 \mathrm{~mm}$ and $\pm 1 / 15 \mathrm{~ms}$, respectively. So the velocity of the bubble is 0.125 $\mathrm{m} / \mathrm{s}$ (uncertainty of $\pm 0.0014 \mathrm{~m} / \mathrm{s}$ ). Similarly, When the fluids in tube 11 flow downwards, the velocity is big with the computed value of $0.346 \mathrm{~m} / \mathrm{s}$ (uncertainty of $\pm 0.0038 \mathrm{~m} / \mathrm{s}$ ) as shown in Fig. 18(b).

Fig. 19 and Fig. 20 give the nadir and peak velocity of fluids in zone I at medium power state, 
respectively. In zone I, two red color cords are located in the adiabatic section as the reference with $\Delta L=100 \mathrm{~mm}$. A bubble in tube 4 is selected as the watch objective, and the velocity behaves slow through the visualization. The time $\Delta t$ passing through the length $\Delta L$ for the bubble head as denoted by the red color circle in Fig. 19 is $0.306 \mathrm{~s}$. So the velocity of the bubble is $0.327 \mathrm{~m} / \mathrm{s}$ (uncertainty of $\pm 0.0016 \mathrm{~m} / \mathrm{s}$ ). Similarly, A bubble in tube 6 is selected as the watch objective in Fig. 20 , the velocity is big through the visualization with the computed value of $0.676 \mathrm{~m} / \mathrm{s}$ (uncertainty of $\pm 0.0034 \mathrm{~m} / \mathrm{s}$ ). Fig. 21 and Fig. 22 give the nadir and peak velocity of fluids in zone I at high power state, respectively. The nadir velocity is computed to $0.454 \mathrm{~m} / \mathrm{s}$ (uncertainty of $\pm 0.0023 \mathrm{~m} / \mathrm{s}$ ), while the peak velocity is computed to $0.83 \mathrm{~m} / \mathrm{s}$ (uncertainty of $\pm 0.0042 \mathrm{~m} / \mathrm{s}$ ).

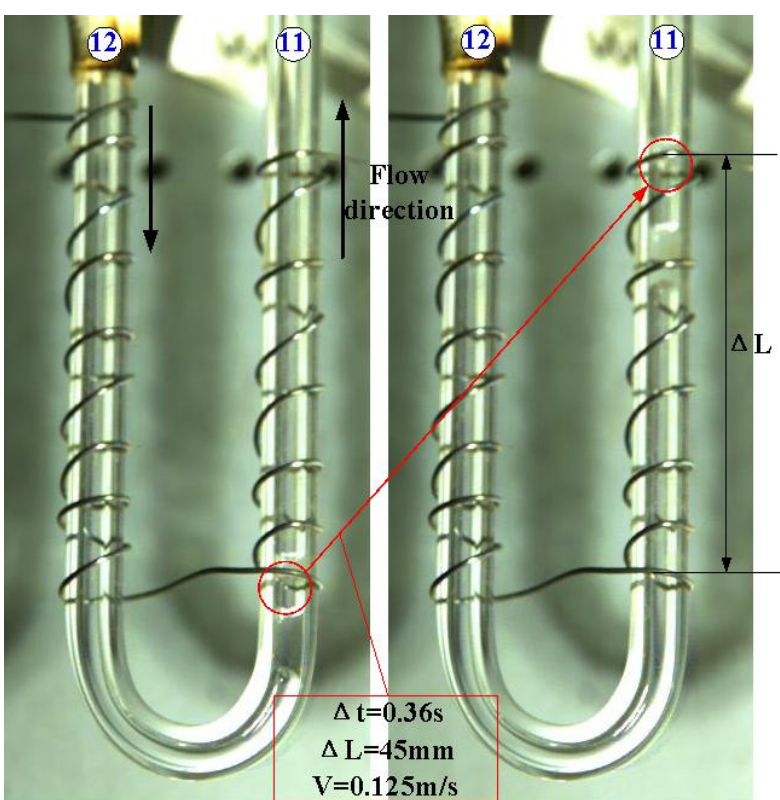

(a)

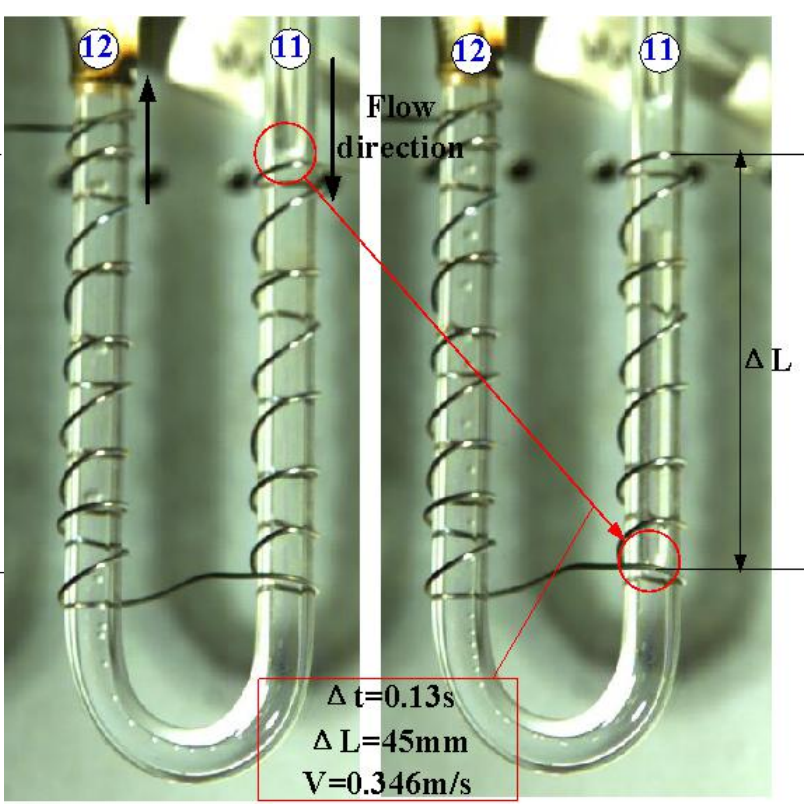

(b)

Fig. 18. The nadir and peak velocity for fluid oscillating at low power state

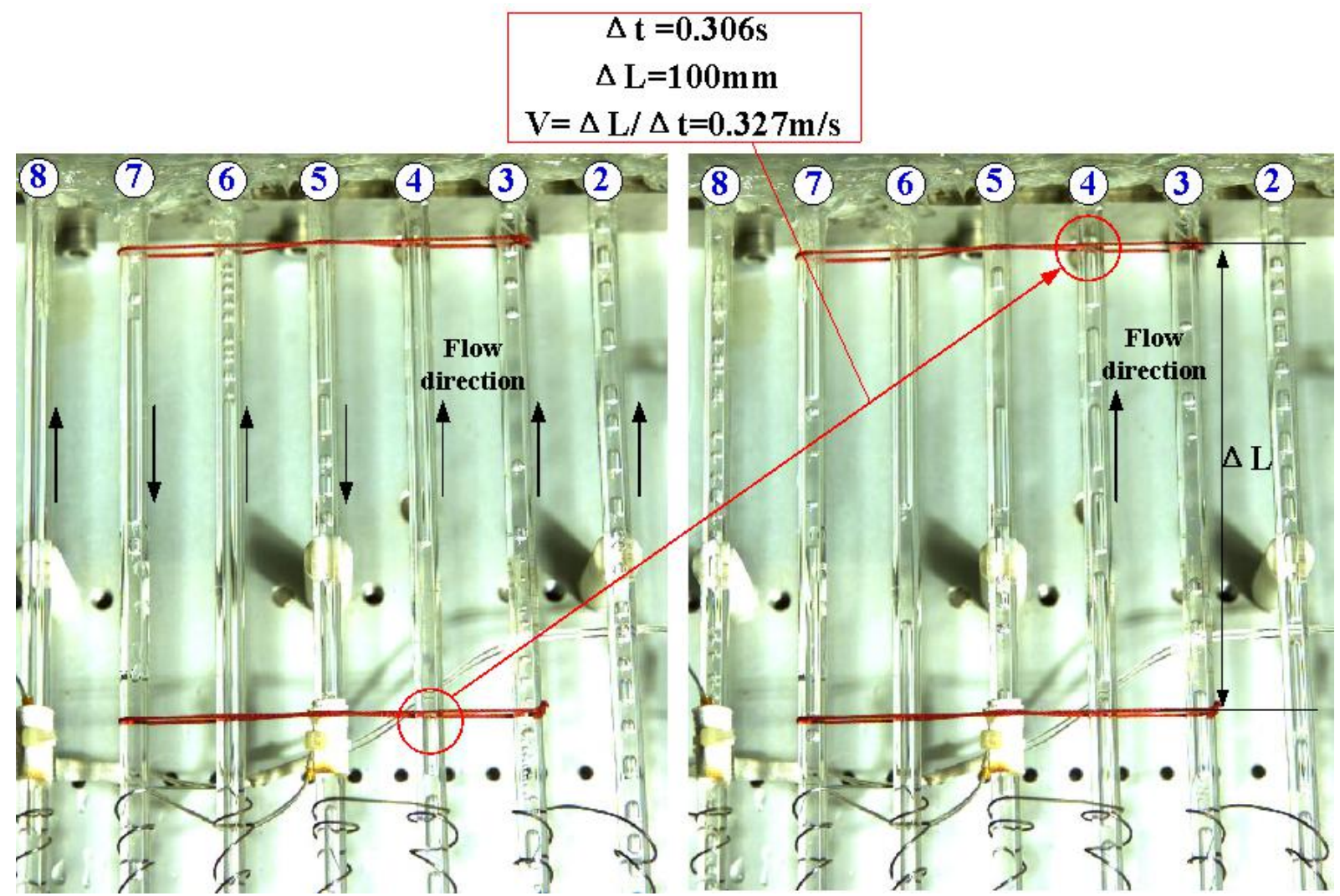


Fig. 19. The nadir velocity for fluid oscillating at medium power state

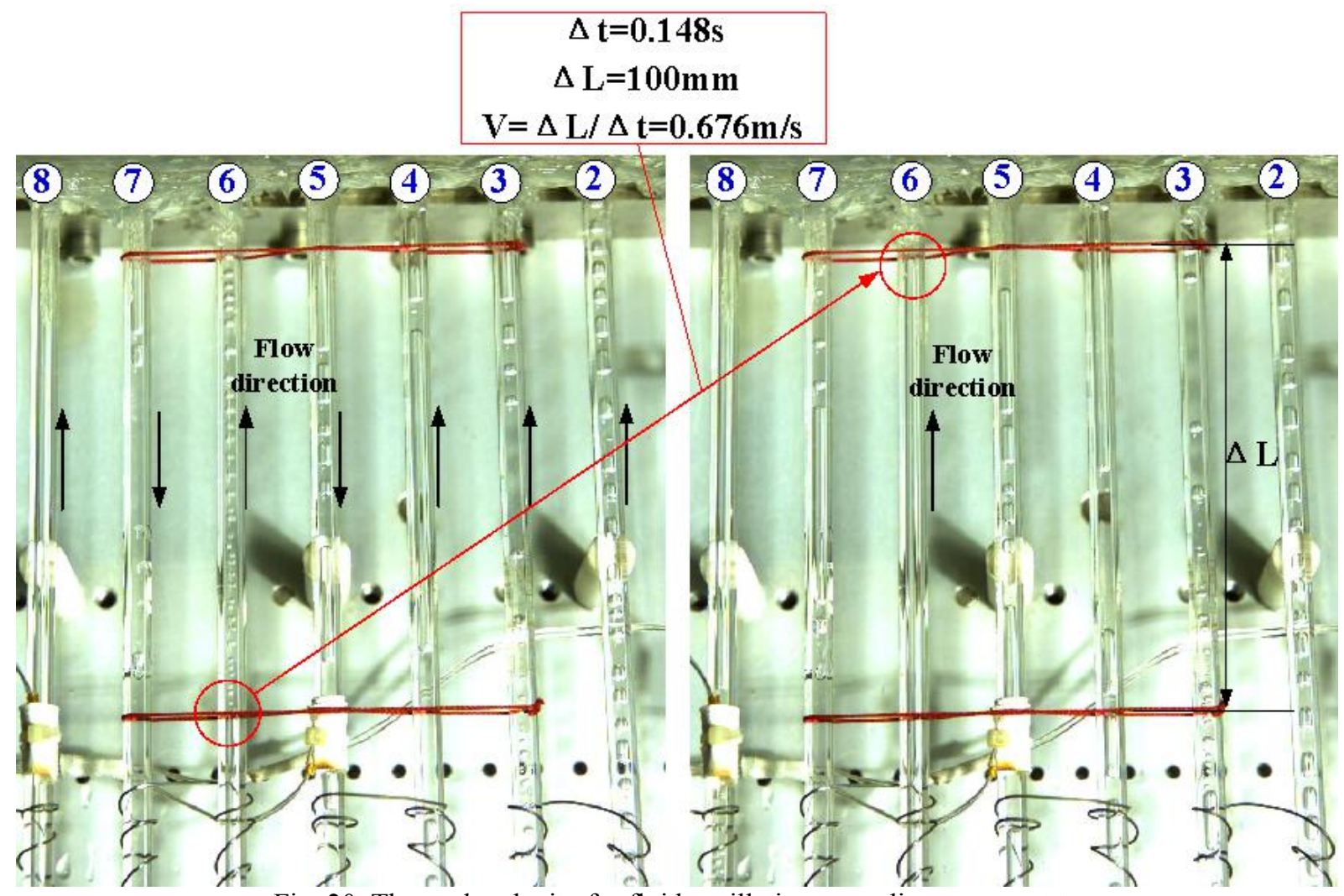

Fig. 20. The peak velocity for fluid oscillating at medium power state

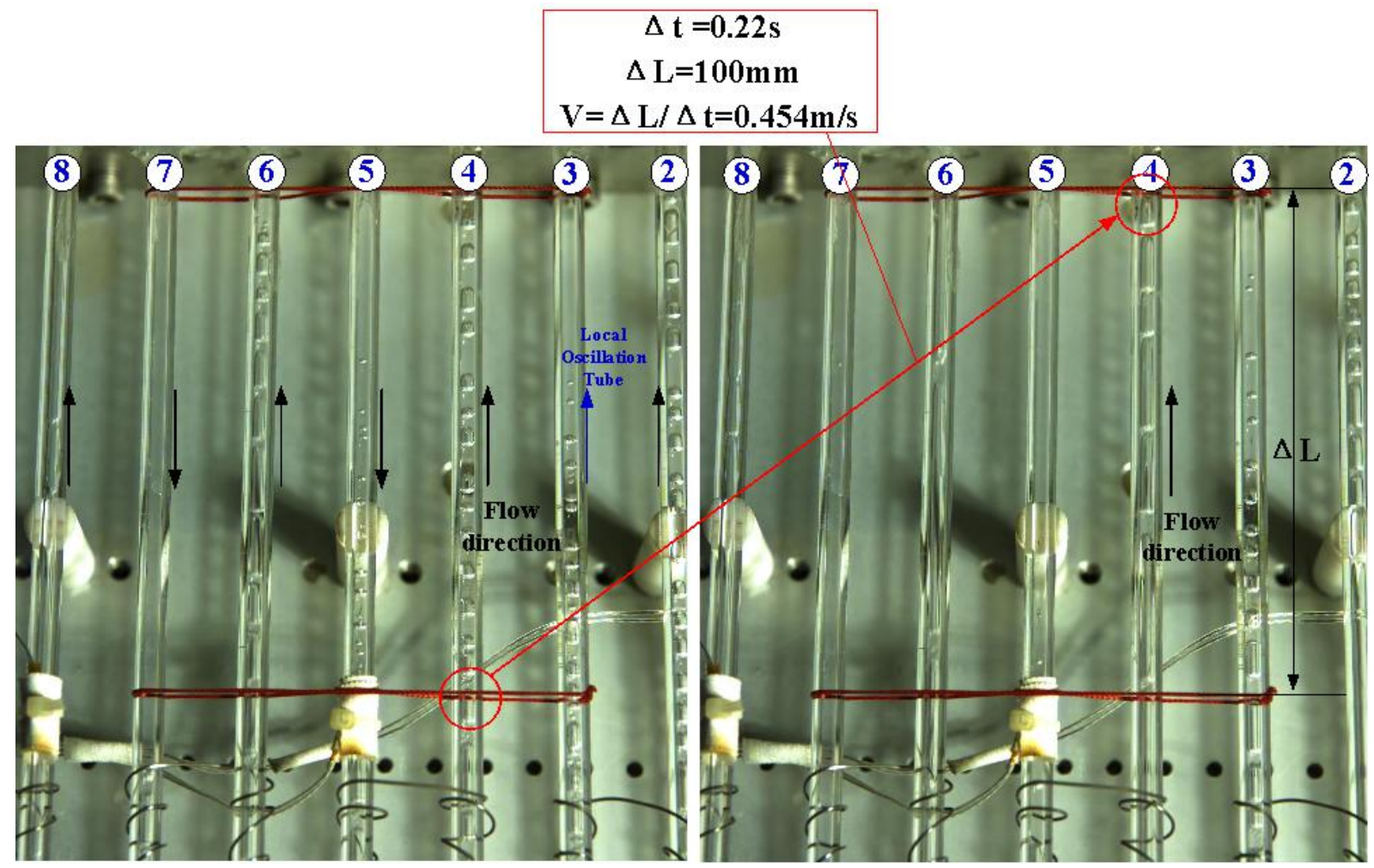

Fig. 21. The nadir velocity for fluid circulation at high power state 


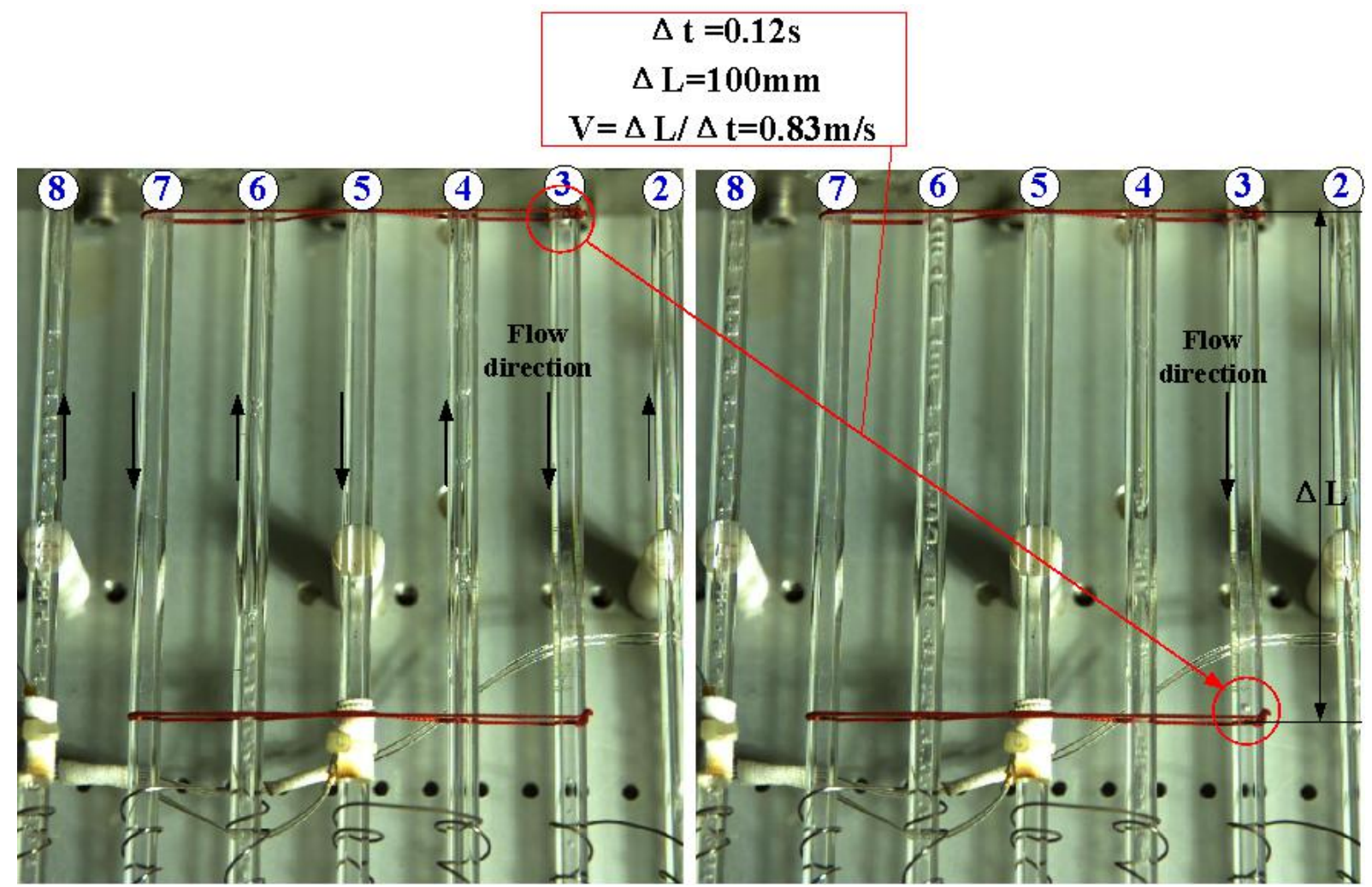

Fig. 22. The peak velocity for fluid circulation at high power state

Based on the full visualization experiment results of ammonia PHP, it is show that, the magnitude of fluid velocity in the tube is about 0.1 to $0.6 \mathrm{~m} / \mathrm{s}$ when the PHP works at the state of "Slug Flow". The magnitude of fluid velocity in the tube is between 0.4 to $0.9 \mathrm{~m} / \mathrm{s}$ when the PHP works at the state of "Annular Flow". Thus, the dimensionless parameters such as Reynolds number (Re), and Capillary number (Ca) can be computed, characterizing the ratio of viscous force and inertia force, the ratio of inertia force and surface tension, respectively. These two parameters can represent for features of fluid flow in PHP, and denote as:

$$
\begin{aligned}
& \operatorname{Re}=\frac{\rho V d}{\mu} \\
& C a=\frac{\mu \cdot V}{\sigma}=\frac{\rho V^{2} d / \sigma}{\rho V d / \mu}=\frac{W e}{\operatorname{Re}}
\end{aligned}
$$

Thus, the Re number ranges from 562.5 to 3375 and $\mathrm{Ca}$ number ranges from $1.054 \mathrm{e}-3$ to $6.327 \mathrm{e}-3$ for "Slug Flow", while the Re number varies from 2733.3 to 6150 and $\mathrm{Ca}$ number varies from 7.850 e-3 to $1.766 \mathrm{e}-2$ for "Annular Flow". It can be concluded that the PHP flow regime is mostly in laminar region for "Slug Flow", but in turbulent region for "Annular Flow". Additionally, the capillary effect could always be occurred due to very low $\mathrm{Ca}$ value no matter what flow pattern is for PHP.

\section{THEORETICAL INVESTIGATION}

\subsection{Mathematical Model}

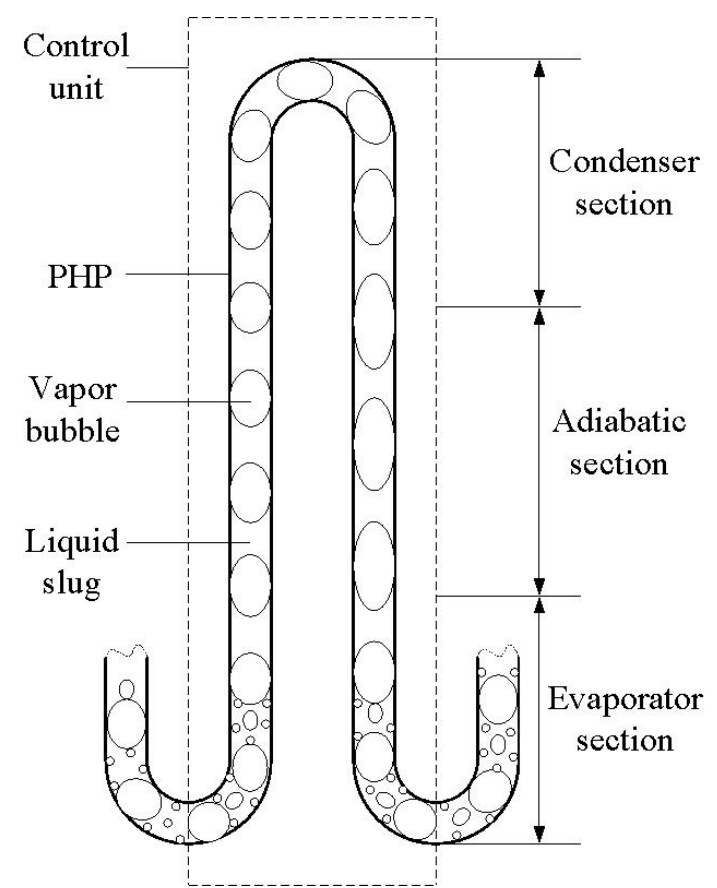

Fig. 23. Schematic of the control unit in PHP model

A schematic of the control unit in PHP model is shown in Fig. 23. The control unit is a typical and 
mirror part of the PHP, including two straight tubes from evaporator to condenser, a full turn in condenser section, a half turn on the left side in evaporator, and another half turn on the right side in evaporator. Obviously, the control unit could contain the whole operating elements of PHP, considering the phase change in evaporator and condenser, the flow pattern variations in motion process, the shear stress and capillary force effects, and the effect of the turns to the flow resistance. It is found that most theoretical models for PHP are oversimplified, assuming that the PHP could be simplified as a straight tube with no turns effect, the flow pattern only refers to the slug flow since the low Reynolds number, and the effects of gravity action and capillary resistance are not considered. Therefore, a new mathematical model in this paper based on the control unit shown in Fig. 23 is contributed without less simplifications.

In order to model heat transfer and flow characteristics in the theoretical model, the following assumptions are made:

(1)The vapor quality in the evaporator and condenser is in linear variation along the flow direction, and no variation in adiabatic section.

(2)Evaporation and condensation heat transfer coefficients are assumed to be constants.

(3)The liquid is incompressible and the vapor is assumed to behave as an ideal gas, both in the saturated state for ammonia working fluid during experiment temperature area.

(4)The heat losses causing by the convection and radiation heat transfer between the ambient to the tube wall of whole PHP are neglected.

\subsection{Governing Equations}

\subsubsection{Conservation Equations}

The momentum equation:

$$
m \frac{d V}{d t}=\sum F=F_{v}-F_{f}-F_{c}-F_{b} \pm F_{g}
$$

where the variables $F_{v}, F_{f}, F_{c}, F_{b}$, and $F_{g}$ represents for the heat driving force from the vapor, the friction resistance of flow, the capillary hysteresis resistance, the drag from the bends and the gravity, respectively.

When PHP operates on a steady or quasi-steady state, the velocity at a fixed point in a tube is not changed a lot as the increment of time for a period at a certain input power. Eventually, the left part of the momentum equation is derived to zero. Thus,

$$
\sum F=F_{v}-F_{f}-F_{c}-F_{b} \pm F_{g}=0
$$

The above equation can be transformed to a pressure relationship,

$$
\Delta P_{v}-\Delta P_{f}-\Delta P_{c}-\Delta P_{b} \pm \Delta P_{g}=0
$$

where the variables $\Delta P_{v}, \Delta P_{f}, \Delta P_{c}, \Delta P_{b}, \Delta P_{g}$ is denoted as the pressure difference or pressure loss resulting by vapor driving force, the friction resistance, capillary hysteresis resistance, the drag from the bends and the gravity, respectively.

The continuity equations:

In evaporator section, for liquid phase and vapor phase, respectively,

$$
\begin{aligned}
& x_{0} \cdot\left(\rho_{l, e} A V\right)+\Delta \dot{m}_{l, e}=x_{1} \cdot\left(\rho_{l, e}^{\prime} A V\right) \\
& \rho_{v, e} \cdot\left[\left(1-\phi_{0}\right) A V\right]+\Delta \dot{m}_{v, e}=\rho_{v, e}^{\prime} \cdot\left[\left(1-\phi_{1}\right) A V\right]
\end{aligned}
$$

In condenser section, for liquid phase and vapor phase, respectively,

$$
\begin{aligned}
& x_{1} \cdot\left[\rho_{l, c} A V\right]+\Delta \dot{m}_{l, c}=x_{0} \cdot\left[\rho_{l, c}^{\prime} A V\right] \\
& \rho_{v, c} \cdot\left[\left(1-\phi_{1}\right) A V\right]+\Delta \dot{m}_{v, c}=\rho_{v, c}^{\prime} \cdot\left[\left(1-\phi_{0}\right) A V\right]
\end{aligned}
$$

where $\phi_{0}$ is the volume flux fraction of liquid phase in entrance of the evaporator section, and $\phi_{1}$ is the volume flux fraction of liquid phase in exit of the evaporator section. While the $x_{0}$ is liquid quality or the mass flux fraction of liquid phase in entrance of the evaporator section, and $x_{1}$ is the mass flux fraction of liquid phase in exit of the evaporator section. The subscripts of $l, v, e, c$ in equations are denoted as the liquid, vapor, evaporator, and condenser, respectively. The superscript apostrophe corresponds to the exit parameters. The variable $\Delta \dot{m}$ is the magnitude of mass flux change, which can be derived from the energy conservation equations.

The relationship between the volume flux fraction and the mass flux fraction can be written as,

$$
x=\frac{\rho_{l} \cdot \phi}{\rho_{l} \cdot \phi+\rho_{v} \cdot(1-\phi)}
$$

and its transformation equation can be written as,

$$
\phi=\frac{\rho_{v} \cdot x}{\rho_{l}(1-x)+\rho_{v} \cdot x}
$$

Because of $\rho_{l} \gg \rho_{v}$ for most working fluids of PHP, the mass flux fraction $x$ is always greater 
than 0.9. So if there is a small variation of $x$, there will be a very large change of $\phi$.

The energy equations:

Heat transfer in PHP is defined as the total heat transfer rate from the heating section to the cooling section, which equals to the total heat input in evaporator if not considering the heat transport losses. Heat transfer could be divided as two part: one part is due to evaporation and condensation phase change of working fluid, and another part is due to heat transfer between the tube wall and fluid in forms of single-phase heat transfer called sensible heat transfer.

In evaporator section, the evaporation heat transfer is,

$$
\Delta \dot{m}_{v, e}=-\Delta \dot{m}_{l, e}=\frac{\beta Q}{h_{f g}}
$$

where $\beta$ is the ratio of evaporative heat to the total heat transport $Q$.

In condenser section, the condensation heat transfer is,

$$
\Delta \dot{m}_{l, c}=-\Delta \dot{m}_{v, c}=\frac{Q_{c}}{h_{f g}}
$$

where $Q_{c}$ is the condensable heat by condensation between the saturated gas to the wall, which can be written as,

$$
Q_{c}=\pi d \cdot L_{c} \cdot h_{s}\left(T_{s}-T_{c}\right)(1-x)
$$

The $h_{s}$ is the average heat transfer coefficient in the condenser, and $L_{c}$ is the length of condenser. The $T_{s}$ is the specific temperature computed as

$$
T_{s}=\left(T_{e}+T_{c}\right) / 2
$$

The sensible heat transfer in evaporator can be calculated by

$$
(1-\beta) Q=\frac{\left(x_{0}+x_{1}\right)}{2} \rho_{l} A \cdot V c_{p}\left(T_{e}-T_{c}\right)
$$

\subsubsection{Condensation}

The value of condensation heat transfer is determined by the two factors, which are condensation heat transfer coefficient $h_{s}$ and vapor quality $x$. While $h_{s}$ has a direct relationship with the velocity of fluid flow in the condenser, and the quality $x$ relates the distribution of liquid and vapor phase. Obviously, these two items are all varied as the flow patterns change.
Prediction of heat transfer during condensation of vapors flowing inside a tube is of great interests for many researchers ${ }^{[37-42]}$. One of the most widely used has been the shah's correlation, covering three flow regimes, such as the Nusselt equation for laminar film condensation in vertical tubes and annular flow condensation correlation.

Fig. 24 gives a schematic of slug flow pattern features at low input power in PHP tube. If the flow pattern is in "Slug Flow", the vapor will flow through the condenser section slowly, and the mechanism of condensation is used following heat transfer equation,

$$
h_{s}=1.32 \operatorname{Re}_{L S}^{-1 / 3}\left[\frac{\rho_{l}\left(\rho_{l}-\rho_{g}\right) g \sin \beta \cdot k_{l}^{3}}{\mu_{l}^{2}}\right]^{1 / 3}
$$

where $\operatorname{Re}_{L S}$ is Reynolds number assuming liquid phase flowing in tube alone.

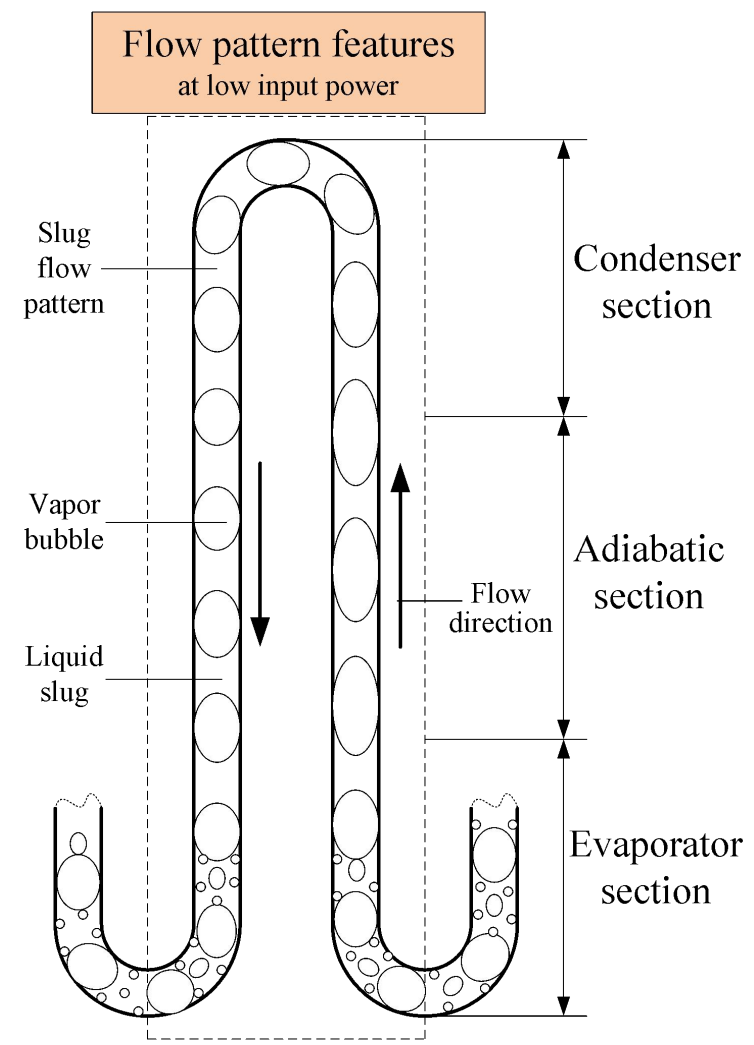

Fig. 24. Flow pattern features of slug flow in PHP

Fig. 25 shows a schematic of annular flow pattern features at high input power in PHP tube. If the flow pattern is in "Annular Flow", the vapor flowing velocity increases, and the vapor bubble becomes long and long, the condensation heat transfer coefficient also grows. In this case, the mechanism of condensation is used with annular flow correlation, 


$$
N u=0.023 \operatorname{Re}_{L S}^{0.8} \operatorname{Pr}_{l}^{0.4}\left[(1-x)^{0.8}+3.8 x^{0.76}(1-x)^{0.04} / P_{r}^{0.38}\right]
$$

$$
h_{s}=N u \cdot k_{l} / d
$$

where the $P_{r}$ is the reduced pressure of vapor in the entrance of condenser.

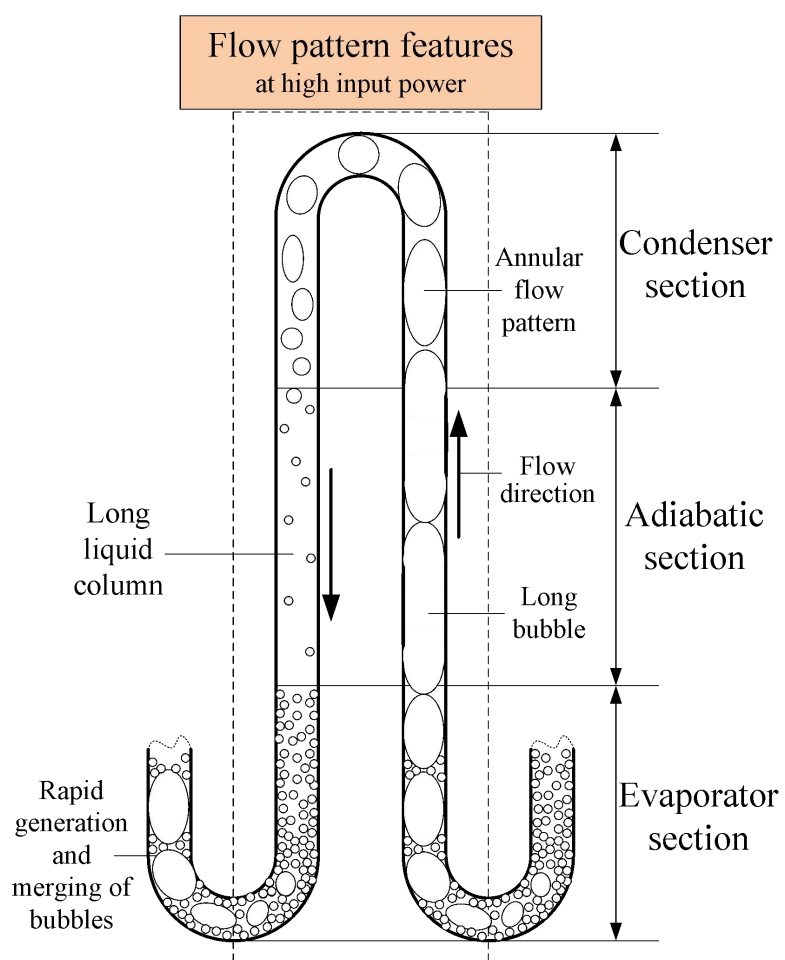

Fig. 25. Flow pattern features of annular flow in PHP

\subsubsection{Pressure Difference or Pressure Loss}

Pressure difference caused by vapor driving force:

When the PHP works at a quasi-steady state, there will be a balance between the driving pressure difference and the pressure loss caused by different drags in the control unit. Fig. 26 illustrates the schematic of flow and heat transfer at quasi-steady state in control unit. Assuming the flow direction of fluid in the tube as shown in the Fig. 26, the entrance and exit of the control unit are defined as the point $a$ and point $b$. The entrance and exit of evaporator section on the right bend are set as the point $a_{0}$ and point $a_{1}$. The exit of condenser section is set as the point $b_{0}$. Thus, the pressure difference between point $a$ and point $b$ is determined as the driving pressure difference, given by:

$$
\Delta P_{v}=p_{a}-p_{b}
$$

The saturated pressure $p_{\text {sat }}$ in the tube of PHP can be derived by the state equation for ideal gas,

$$
p_{s a t}=\rho_{v} \cdot R \cdot T
$$

At the position of point a, the density of vapor can be obtained using the conservation equation (8) trough the point $\mathrm{a}_{0}$, as:

$$
\rho_{a}\left[\left(1-\phi_{a}\right) A V\right]=\rho_{a 0} \cdot\left[\left(1-\phi_{0}\right) A V\right]+\frac{\beta Q / 2}{h_{f g}}
$$

where $\phi_{a}$ is the volume flux fraction of liquid phase at point $a_{0}$, which could be computed from the mass flux fraction $x_{a}$. The initiative value of vapor density at point $\mathrm{a}_{0}$, is given according to the flow patterns. If the flow pattern of PHP is in "Slug Flow", it equals to the saturated vapor density corresponding to the saturated temperature at the exit of condenser. If the flow pattern of PHP is in "Annular Flow", it can be neglected as very small value due to rare vapor bubbles under condensation from condenser section.

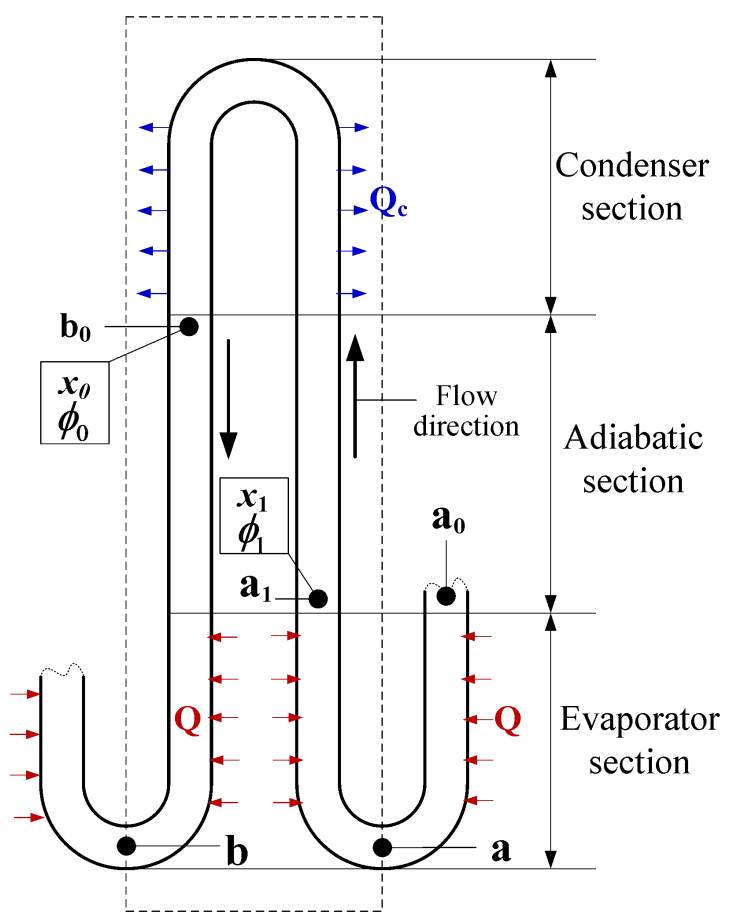

Fig. 26. Schematic of flow and heat transfer in control unit

The $x_{a}$ can be computed by the conservation equation (7), as:

$$
x_{a}=x_{0}-\frac{\beta Q / 2}{\rho_{l} A V h_{f g}}
$$


In a similar way, the density of vapor at the point $\mathrm{a}_{1}$ could be derived as,

$$
\rho_{a 1}\left[\left(1-\phi_{1}\right) A V\right]=\rho_{a} \cdot\left[\left(1-\phi_{a}\right) A V\right]+\frac{\beta Q / 2}{h_{f g}}
$$

The density of vapor at the point $b_{0}$ could be yielded as,

$$
\rho_{a 1}\left[\left(1-\phi_{1}\right) A V\right]-\frac{Q_{c}}{h_{f g}}=\rho_{b 0} \cdot\left[\left(1-\phi_{0}\right) A V\right]
$$

The density of vapor at the point $b$ could be yielded as,

$$
\rho_{b 0}\left[\left(1-\phi_{0}\right) A V\right]+\frac{\beta Q / 2}{h_{f g}}=\rho_{b} \cdot\left[\left(1-\phi_{b}\right) A V\right]
$$

Therefore, the driving pressure difference by heat transfer is given by,

$$
\Delta P_{v}=p_{a}-p_{b}=\left(\rho_{a}-\rho_{b}\right) R T_{e}
$$

Pressure loss caused by friction resistance:

$$
\Delta P_{f}=\frac{4 \cdot C_{f}}{d} \cdot l \cdot \frac{\rho_{l} V^{2}}{2}
$$

where the friction coefficient is determined by:

$$
C_{f}=\left\{\begin{array}{lc}
16 / \mathrm{Re} & \mathrm{Re} \leq 1180 \\
0.078 \mathrm{Re}^{-0.2} & 1180<\operatorname{Re}<12000 \\
0.057 \mathrm{Re}^{-0.2} & 12000 \leq \mathrm{Re}<1 \mathrm{e}+7
\end{array}\right.
$$

Pressure loss caused by capillary hysteresis resistance:

When the vapor bubble flows in a capillary, the interface curve between the liquid and vapor phase will be changed, leading that the advancing contact angle which locates in the head of the vapor is a bit smaller than the static contact angle, while the receding contact angle which locates in the end of the vapor is slightly bigger than the static contact angle. The change of contact angle for liquid slugs is absolutely opposite to the change for vapor bubbles. These variations are not beneficial for liquid slugs motion, forming a moving drag named by capillary hysteresis resistance.

In a capillary, the capillary suction force $P_{c}$ is determined by the Young-Laplace equation,

$$
P_{C}=\frac{4 \sigma \cdot \cos \theta^{\prime}}{d}
$$

where $\sigma$ is the facial tension at the interface, $\theta^{\prime}$ is the contact angle.
Thereby, the pressure loss caused by capillary hysteresis resistance for liquid slugs is

$$
\begin{aligned}
\Delta P_{c}= & \sum_{1}^{N}\left(P_{c, r e c}-P_{c, a d v}\right)= \\
& \sum_{1}^{N} \frac{4}{d}\left(\sigma_{r e c} \cos \theta_{r e c}^{\prime}-\sigma_{a d v} \cos \theta_{a d v}^{\prime}\right)
\end{aligned}
$$

where $N$ denotes the number of the liquid slugs which is of great different for different flow patterns, acquired by the full visualization test.

When the capillary number is small, for example $C a<0.1$, Beithier et al. ${ }^{[43]}$ simplifies the expression form of the advancing contact angle and the receding contact angle, as:

$$
\begin{aligned}
& \theta_{a d v}^{\prime}=\theta_{s}^{\prime}+\frac{\text { const } \cdot|C a|}{3 \theta_{s}^{\prime 2}} \\
& \theta_{\text {rec }}^{\prime}=\theta_{s}^{\prime}-\frac{\text { const } \cdot|C a|}{3 \theta_{s}^{\prime 2}}
\end{aligned}
$$

where $\theta_{s}^{\prime}$ is static contact angle, and the constant equals to 94 .

Pressure loss caused by the bends:

When the number of turns/bends and the magnitude of velocity increase, the pressure losses related to the turns/bends in a PHP could not be neglected and must be taken into consideration. There are two kinds of bends, as shown in Fig. 27.
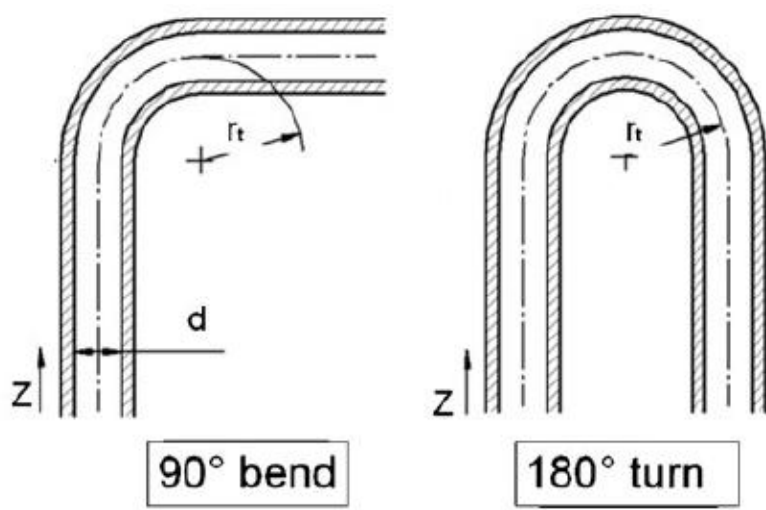

Fig. 27 Geometry of $90^{\circ}$ bend and $180^{\circ}$ turn in PHP

The pressure loss caused by the bends can be written as,

$$
\Delta P_{b}=\sum_{1}^{n} K_{k} \frac{l}{d} \cdot \frac{\rho_{l} V^{2}}{2}
$$

where the parameter $K_{k}$ is the bend loss coefficient, which can be applicable to the $3-\mathrm{K}$ correlation given by Darby ${ }^{[44,45]}$, 


$$
K_{k}=\frac{K_{\mathrm{Re}}}{\operatorname{Re}_{l}}+K_{l}\left(1+\frac{K_{d}}{(d / 0.0254)^{0.3}}\right)
$$

This empirical correlation depends on three main parameters ( $\left.K_{\mathrm{Re}}, K_{l}, K_{d}\right)$, on the Reynolds number and on the exact geometry of the bend. The 3-K constants for loss coefficients for different bends are listed in Table 1.

Pressure loss caused by the gravity:

When PHP works in "Slug Flow", the liquid slugs and vapor bubbles are in oscillating motion. In this case, the pressure loss caused by the gravity sometimes is positive behaving as the driving force, sometimes is negative acting as the drag force. While PHP works in "Annular Flow", there is a one-direction circulation forming, and the gravity always plays as a driving force. In other words, the gravity is beneficial for the PHP operating. But how much is the effect of gravity, none of the existing models detailed this phenomenon.

The pressure loss caused by the gravity is

$$
\Delta P_{g}= \pm\left[\begin{array}{l}
\rho \mathrm{gL}_{\mathrm{a}} \cdot \sin \theta \cdot\left(\mathrm{x}_{0}-\mathrm{x}_{1}\right)+ \\
\rho \mathrm{g}\left(\mathrm{L}_{\mathrm{c}}+\mathrm{L}_{\mathrm{h}}+\pi \cdot \mathrm{r}_{t}\right) \cdot\left(\mathrm{x}_{0}-\mathrm{x}_{1}\right) / 2
\end{array}\right]
$$

where $L_{h}$ and $L_{a}$ are the length of evaporator and adiabatic section, respectively. The item $r_{t}$ is the radius of the turn of PHP.

Table 1. 3-K constants for loss coefficients.

\begin{tabular}{c|c|c|c|c}
\hline Fitting type & $r_{t} / d$ & $K_{\mathrm{Re}}$ & $K_{l}$ & $K_{d}$ \\
\hline Bend $90^{\circ}$ & 1 & 800 & 0.091 & 4.0 \\
\cline { 2 - 5 } & 1.5 & 800 & 0.071 & 4.2 \\
\cline { 2 - 5 } & 2 & 800 & 0.056 & 3.9 \\
\cline { 2 - 5 } & 4 & 800 & 0.066 & 3.9 \\
\cline { 2 - 5 } & 6 & 800 & 0.075 & 4.2 \\
\hline Turn $180^{\circ}$ & 1 & 1000 & 0.12 & 4.0 \\
\cline { 2 - 5 } & 1.5 & 1000 & 0.1 & 4.0 \\
\cline { 2 - 5 } & 2.5 & 1000 & 0.1 & 4.0 \\
\hline
\end{tabular}

\subsubsection{Transformation Relations}

Combining equations (7) and (13) can yields,

$$
\Delta x=x_{0}-x_{1}=\frac{\beta Q}{\rho_{l e} A V \cdot h_{f g}}
$$

Substituting equation (38) into equation (17), obtains:

$$
\begin{aligned}
(1-\beta) Q+ & \frac{\beta Q}{2} \cdot \frac{\rho_{l}}{\rho_{l e}} \frac{c_{p}\left(T_{e}-T_{c}\right)}{h_{f g}} \\
& =x_{0} \cdot \rho_{l} A \cdot V c_{p}\left(T_{e}-T_{c}\right)
\end{aligned}
$$

Defining $W_{1}=\rho_{l} A \cdot h_{f g}$, on behalf of the latent heat transfer, and $W_{2}=\rho_{l} A \cdot c_{p}\left(T_{e}-T_{c}\right)$, expressing the sensible heat transfer, thus, the equation (38) can be turned into,

$$
\Delta x=x_{0}-x_{1}=\left(\beta \cdot \frac{\rho_{l}}{\rho_{l e}}\right) \frac{Q}{V \cdot W_{1}}
$$

The equation (39) can be transformed as,

$$
(1-\beta) Q+\frac{\beta Q}{2} \cdot \frac{\rho_{l}}{\rho_{l e}} \frac{W_{2}}{W_{1}}=x_{0} \cdot W_{2} \cdot V
$$

Thereby, the velocity of fluids at quasi-steady state in PHP can be computed as,

$$
V=\frac{E_{1}+E_{2}}{E_{3}}
$$

where,

$$
\begin{aligned}
& E_{1}=(1-\beta) Q \\
& E_{2}=\frac{\beta Q}{2} \cdot \frac{\rho_{l}}{\rho_{l e}} \frac{W_{2}}{W_{1}} \\
& E_{3}=x_{0} \cdot W_{2}
\end{aligned}
$$

It is noted that the velocity is main related with the total heat transfer $Q$, the $\beta$, the fluid temperature of evaporator and condenser as $T_{e}$ and $T_{c}$, and the mass flux fraction of liquid phase in entrance $x_{0}$.

\subsection{Analytical Results}

\subsubsection{Effect Mechanism of Operating Parameter}

In order to investigate the effect of main operating parameter to the velocity, the first step is to require the fluids temperature of evaporator and condenser as $T_{e}$ and $T_{c}$ in the tube of PHP. 
Obviously, the level of $T_{e}$ and $T_{c}$ is determined by the total heat transfer $Q$, as shown in Fig.7 to Fig. 9. However, the temperature measured in the experiment is the temperature of outside wall of the tubes in Fig. 7 to Fig. 9. The temperature of inner wall of the tubes should be of a conversion using the thermal resistance of tube wall, as

$$
R=\frac{\ln \left(d^{\prime} / d\right)}{2 \pi \lambda \cdot l}
$$

where $\lambda$ is the heat conductance of the quartz glass.

Fig. 28 shows the revised temperature of $T_{e}$ and

$T_{c}$ under different power based on the measured temperature. In the figure, the black curve gives an average value of evaporator outlet temperature, and the red curve provides an average temperature of condenser in the experiment. The blue curve gives the revised temperature of $T_{e}$ corresponding to the black curve, and the green curve shows the revised temperature of $T_{c}$ corresponding to the red curve.

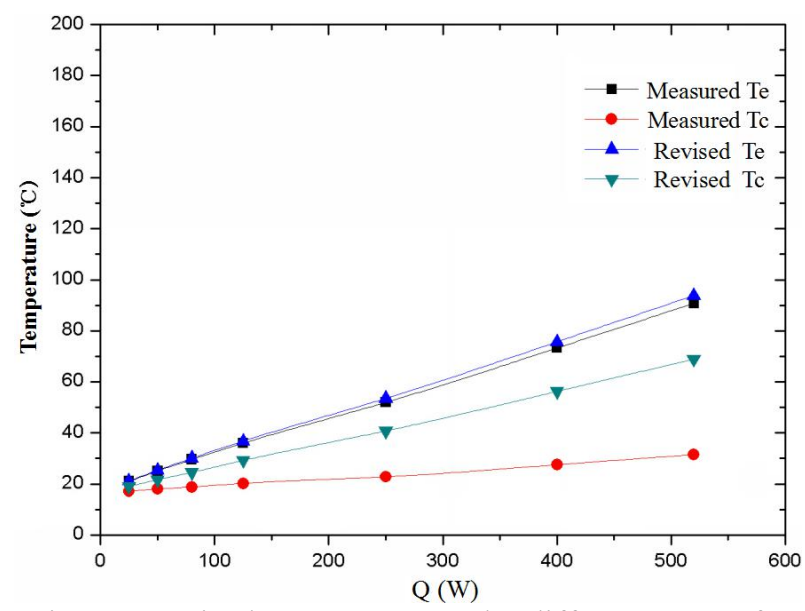

Fig. 28. Revised temperature under different power for Te and $\mathrm{Tc}$

Fig. 29 shows the effect of $\beta$ on the value of $E_{2} / E_{1}$ under different power. It is found that, no matter whether PHP works at low power or high power mode, if $\beta$ ranges from 0.1 to 0.5 , the ratio value of $E_{2} / E_{1}$ is always lower that 0.04 for ammonia working fluid. In other words, if $\beta \leq 0.5$, there will be a relationship of $E_{2} \ll E_{1}$. Therefore, the equation (42) can be simplified as

$$
V \approx \frac{E_{1}}{E_{3}}=\frac{(1-\beta) Q}{x_{0} \cdot \rho_{l} A c_{p}\left(T_{e}-T_{c}\right)}
$$

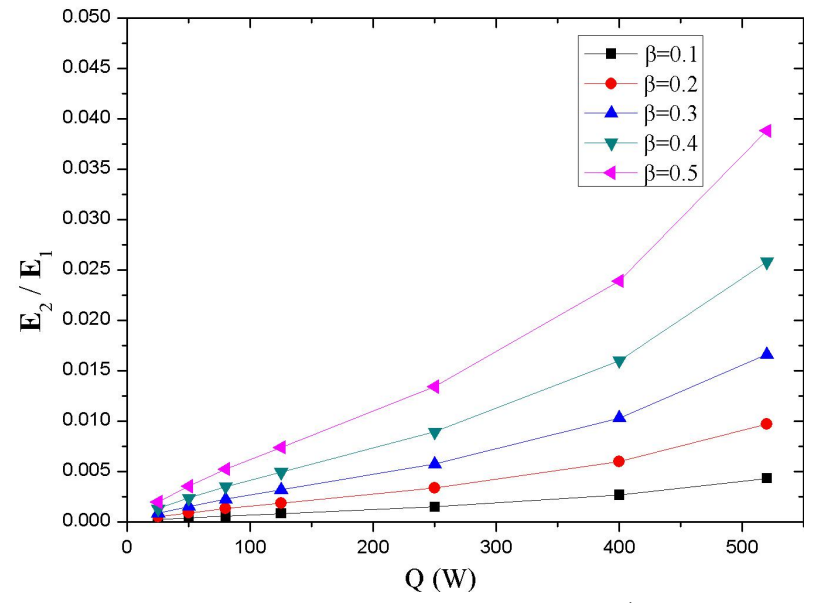

Fig. 29. Effect of $\beta$ on the value of $E_{2} / E_{1}$ under different power

The effect of $\beta$ and $x_{0}$ on the flow velocity under different power is shown in Fig. 30 by computing the equation (47). The results show that the velocity of fluids in ammonia PHP increases continuously as the increment of transport power, but more and more slowly due to increment of $\beta$ and temperature difference between $T_{\mathrm{e}}$ and $T_{\mathrm{c}}$. When the ratio of evaporative heat to the total heat transfer defined as $\beta$ decreases, the velocity will increase rapidly, meaning that the velocity reflects the increment of sensible heat transfer of the PHP. However, on the other hand, if the $\beta$ decreases, the phase change of evaporation will decrease, resulting that the driving force to the fluids in PHP decreases simultaneously, and velocity of fluids becomes small. It is a contradictory relation, thereby there will be a balanced value of $\beta$ e.g. 0.2 to 0.3 , for optimal working performance at a given transport power. This analysis result could explain that in PHP operating mechanism, the latent heat compared to sensible heat is small, but it is important as a driving role on PHP.

The mass flux fraction of liquid phase in entrance of the evaporator section defined as $x_{0}$ also plays a notable impact on the velocity as shown in Fig. 30. The $x_{0}$ represents the flow pattern features in PHP. When the flow pattern changes from slug flow to annular flow, the value of $x_{0}$ will increase. From the figure, it is seen that as the $x_{0}$ increases, the velocity will decrease. This phenomenon also can explain that the velocity will not increase rapidly step by step as the total transport power increases, due to the flow pattern transit.

Fig. 31 shows the effect of $\beta$ and $x_{0}$ on the $\Delta x$ under different power. The increment of $\beta$ and 
total transport power have significant impact on the $\Delta x$. The difference between $x_{0}$ to $x_{1}$ defined as $\Delta x$ will grows explosively like a exponential function as the $\beta$ and total input power increase. From equation (12), if there is a small variation of $x$ defined as $\Delta x$, there will be a very large change of $\phi$ named as $\Delta \phi$. Obviously, the change of volume flux fraction will be more violent than the mass flux fraction of PHP as the power increases. This is the reason that as the transport power increases, the flow pattern in PHP will change largely. However, the $\Delta x$ is not very sensitive with the variation of $x_{0}$ as shown in Fig. 31.

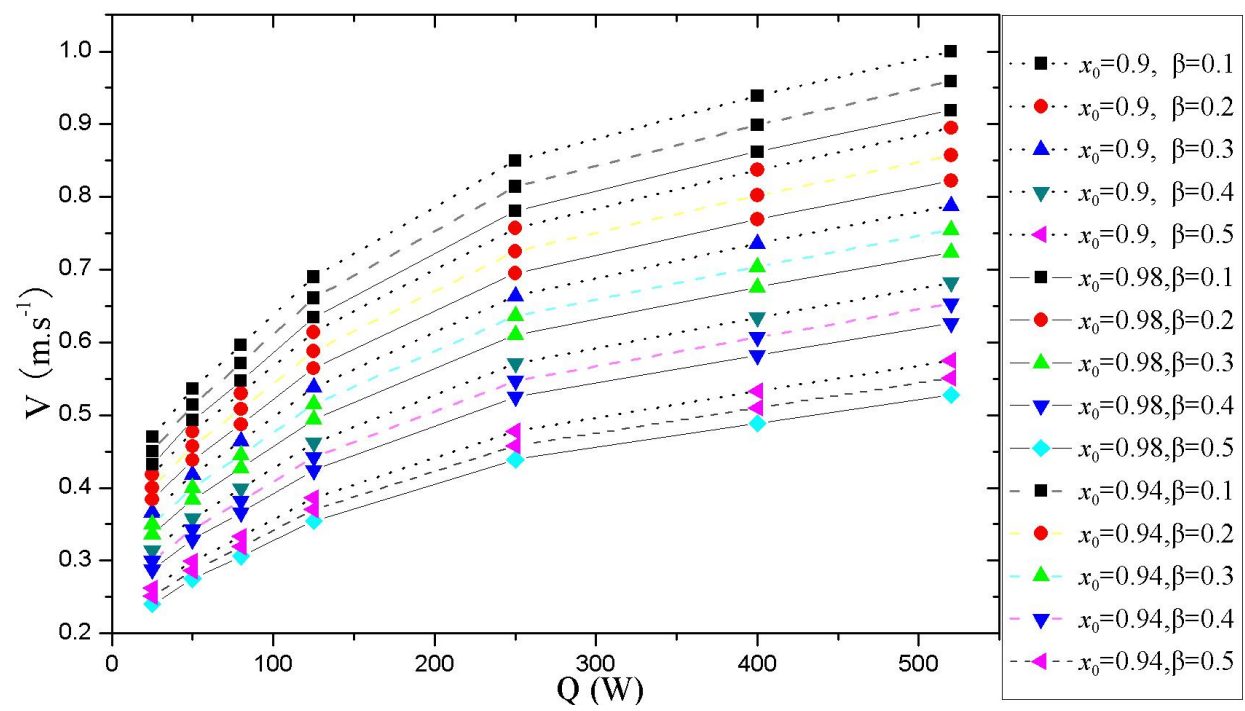

Fig. 30 Effect of $\beta$ and $x_{0}$ on the flow velocity under different power

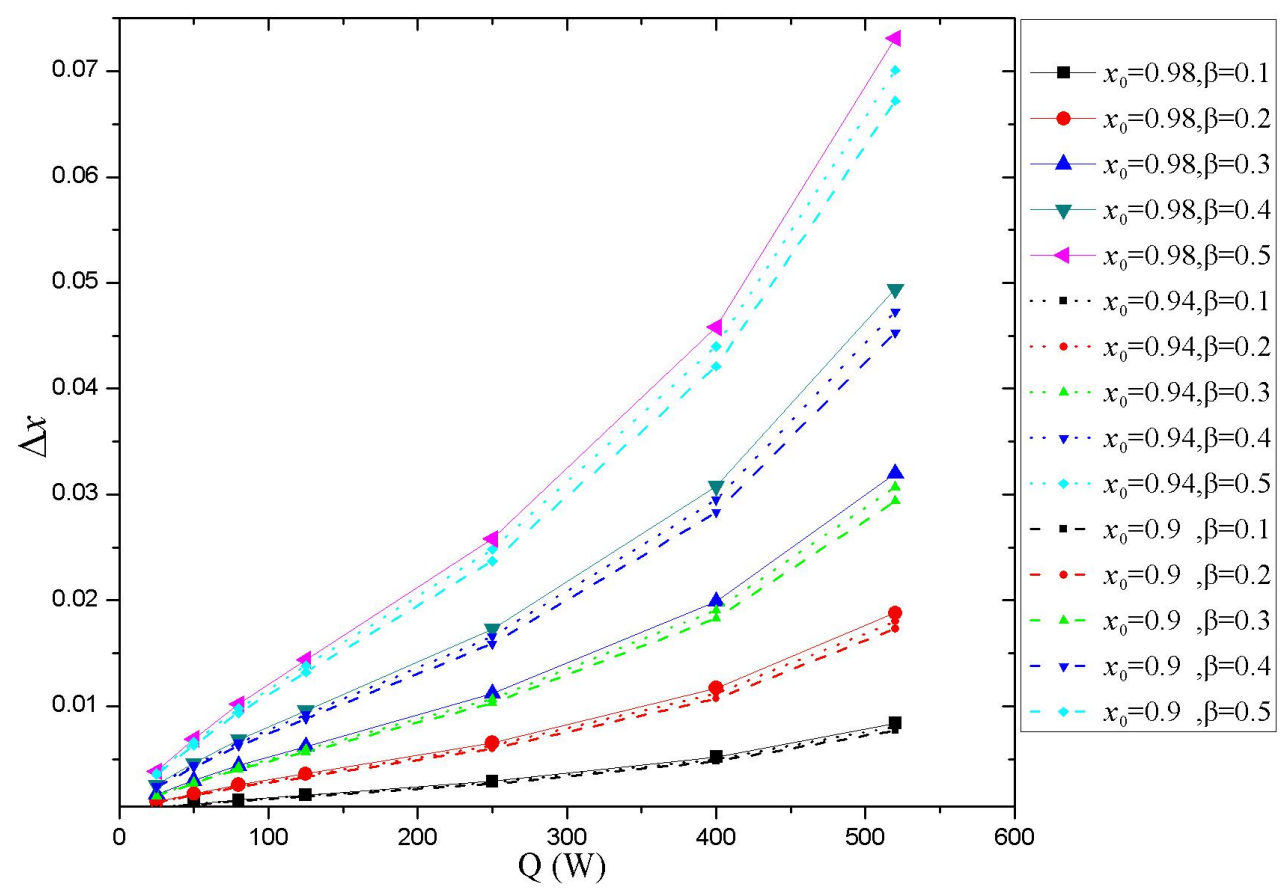

Fig. 31 Effect of $\beta$ and $x_{0}$ on the $\Delta x$ under different power

Table 2 Basic parameter of PHP in analytical computation

\begin{tabular}{c|c|c|c|c|c|c|c}
\hline Parameter & turns & $L_{a}(\mathrm{~m})$ & $L_{e}(\mathrm{~m})$ & $L_{c}(\mathrm{~m})$ & $\begin{array}{c}\text { External tube } \\
\text { diameter }(\mathrm{mm})\end{array}$ & $\begin{array}{c}\text { Internal tube } \\
\text { diameter }(\mathrm{mm})\end{array}$ & $\begin{array}{c}\text { Filling } \\
\text { ratio }\end{array}$ \\
\hline Value & 6 & 0.12 & 0.1 & 0.1 & 6 & 2 & $70 \%$ \\
\hline
\end{tabular}

Table 3 Iteration computation results of flow and heat transfer identities 


\begin{tabular}{c|c|c|c|c|c|c|c|c}
\hline $\begin{array}{c}\text { Power } \\
(\mathrm{W})\end{array}$ & $\beta$ & $\begin{array}{c}V \\
(\mathrm{~m} / \mathrm{s})\end{array}$ & $\phi_{0}$ & $\begin{array}{c}\Delta P_{v} \\
(\mathrm{~Pa})\end{array}$ & $\begin{array}{c}\Delta P_{f} \\
(\mathrm{~Pa})\end{array}$ & $\begin{array}{c}\Delta P_{c} \\
(\mathrm{~Pa})\end{array}$ & $\begin{array}{c}\Delta P_{g} \\
(\mathrm{~Pa})\end{array}$ & $\begin{array}{c}\Delta P_{b} \\
(\mathrm{~Pa})\end{array}$ \\
\hline 25 & 0.077 & 0.21 & 0.68 & 6111 & 1626 & 138 & 1640 & 2703 \\
\hline 80 & 0.144 & 0.51 & 0.64 & 19108 & 7807 & 330 & 1491 & 9466 \\
\hline 520 & 0.324 & 0.70 & 0.91 & 23062 & 9547 & 396 & 301 & 12920 \\
\hline
\end{tabular}

\subsubsection{Flow and Heat Transfer identities analysis}

In order to obtain the flow and heat transfer identities of PHP at quasi-steady state, all the conservation equations including equation (4) to equation (17) are combined to have an analytical balanced solution by an iteration method. The inclination angle is $60^{\circ}$, and three transport powers of $25 \mathrm{~W}, 80 \mathrm{~W}$, and $520 \mathrm{~W}$ are selected, on behalf of low, medium, and high power state, respectively. For comparison of theoretical results and experimental results, the same parameters are used like experiment in analytical computation as given in Table 2.

The main parameters representing the flow and heat transfer identities of ammonia PHP, such as velocity, $\beta, \phi_{0}, \Delta P_{v}, \Delta P_{f}, \Delta P_{c}, \Delta P_{b}$, and $\Delta P_{g}$, are computed at three different power, shown in Table 3 .

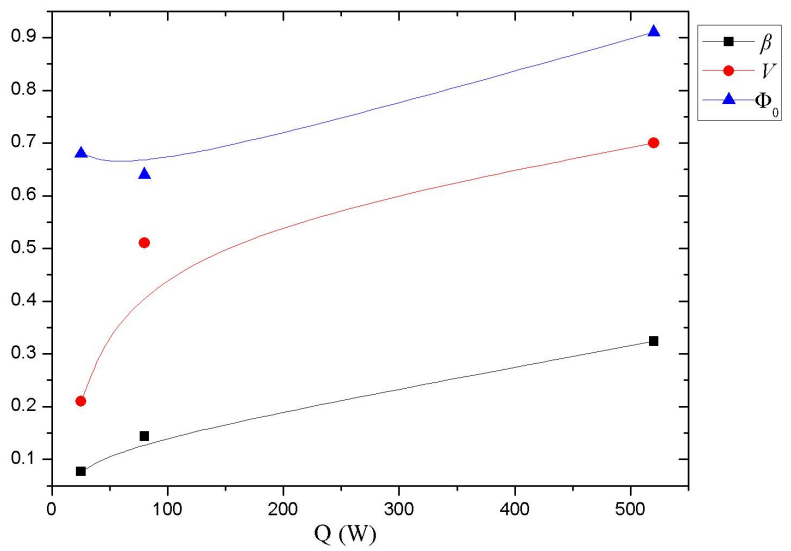

Fig. 32. Variation of $\beta, V$ and $\phi_{0}$ as total power increment

Fig. 32 shows the variation of $\beta, V$ and $\phi_{0}$ as the power increases. It is found that the ratio of evaporative heat to the total heat input increases from 0.077 to 0.324 as total transport power increases from $25 \mathrm{~W}$ to $520 \mathrm{~W}$. The velocity of fluids in tube is $0.21 \mathrm{~m} / \mathrm{s}, 0.51 \mathrm{~m} / \mathrm{s}$ and $0.7 \mathrm{~m} / \mathrm{s}$ when the input power is $25 \mathrm{~W}, 80 \mathrm{~W}$ and $520 \mathrm{~W}$, respectively. Obviously, the theoretical velocity results are good agreement with the experiment results at different transport power. The volume flux fraction of liquid phase in entrance of the evaporator section defined as $\phi_{0}$ changes largely from 0.64 to 0.91 when the power increases from $80 \mathrm{~W}$ to $520 \mathrm{~W}$, indicated that the flow pattern in tube is of large variation at high input power. It is demonstrated that theoretical prediction results of flow pattern agree well with the practical operating state of ammonia PHP.

The theoretical computing results also can be obtained the relationship between the driving pressure difference and different pressure loss items. From the table 3, it is observed that the maximum pressure loss item is caused by the bends. In order to research the contributions of capillary force and gravity for PHP operating at different input power, the ratio of pressure loss caused by capillary resistance to pressure loss caused by the bends is defined as $\varepsilon$, and the ratio of pressure difference caused by gravity to pressure loss caused by the bends is defined as $\eta$.

$$
\begin{gathered}
\varepsilon=\frac{\Delta P_{c}}{\Delta P_{b}} \\
\eta=\frac{\Delta P_{g}}{\Delta P_{b}}
\end{gathered}
$$

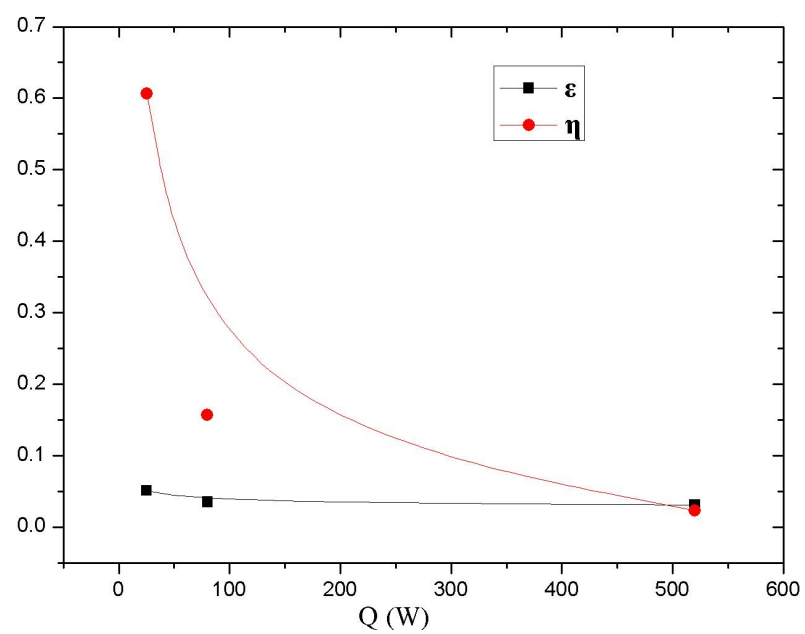

Fig. 33. Variation of $\varepsilon$ and $\eta$ as total power increment

Fig. 33 shows the variation of $\varepsilon$ and $\eta$ as power increases. It is seen that the $\varepsilon$ is very low, and decreases a little as the power increases, indicating that the pressure loss cause by capillary resistance is small refer to other items. The gravity effect to the flow performance of PHP is dramatic at low 
power state as the red curve shown in Fig. 33. However, it decreases rapidly as the input power increases and could be neglected, particularly when the flow pattern changes into annular flow. From the experiment results, it is found that the gravity has a little benefit for the circulation of PHP.

\section{CONCLUSIONS}

A new full visualization experiment of ammonia PHP with high speed camera is conducted, to study the relationship between the flow patterns and thermo-hydrodynamics of the PHP. At the same time, a theoretical model of heat transfer and flow mechanism coupled different flow patterns is proposed, and the results are compared with experiment results. The following conclusions are obtained:

(1) The flow pattern is in "Slug Flow", when PHP operates at low power state, for example, lower than $80 \mathrm{~W}$ for 6 turns of ammonia PHP. While the PHP works at high power state, the flow pattern in tube will transform into "Annular Flow", and a single one-direction circulation is formed.

(2) The accurate velocity in tube of practical ammonia PHP is firstly captured by visualization experiment at different flow patterns. The magnitude of fluid velocity is about 0.1 to $0.6 \mathrm{~m} / \mathrm{s}$ when ammonia PHP works at the state of "Slug Flow". And the magnitude of fluid velocity is between 0.4 to $0.9 \mathrm{~m} / \mathrm{s}$ when the PHP works at the state of "Annular Flow".

(3) Flow regime of PHP could be regarded as laminar working region in "Slug Flow", due to Re number ranging from 562.5 to 3375 for ammonia PHP, but working at turbulent region in "Annular Flow", with Re number ranging from 2733.3 to 6150. Moreover, the capillary effect could always be occurred due to very low $\mathrm{Ca}$ value no matter what flow pattern is.

(4) The theoretical results show that the velocity and flow pattern in tube are good agreement with the experiment results. The volume flux fraction of liquid phase in entrance of the evaporator section changes to 0.91 when the transport power increases to $520 \mathrm{~W}$, well agreed with the practical observation in experiment.

(5) The ratio of evaporative heat to the total heat input increases from 0.077 to 0.324 as the power increases from $25 \mathrm{~W}$ to $520 \mathrm{~W}$. The results could explain that, for PHP operating mechanism, the latent heat compared to sensible heat is small, but it is important as a driving role on PHP.
(6) The gravity effect to the flow performance of PHP is dramatic at low power state. However, it decreases rapidly and could be neglected, as the flow pattern of PHP changes into "Annular Flow".

(7) For PHP, its flow and heat transfer performance have a direct communication with its flow pattern features. In "Annular Flow", a single one-direction circulation with very high heat transfer performance occurred. Therefore, it could be predicted that the PHP will have better performance no matter in space or at ground, if the flow pattern of PHP is enforced towards "Annular Flow" with some artificial controls or methods.

\section{ACKNOWLEDGEMENT}

The authors gratefully acknowledge financial support for this work from the National Science Foundation of China (NSFC 51176190).

\section{NOMENCLATURE}

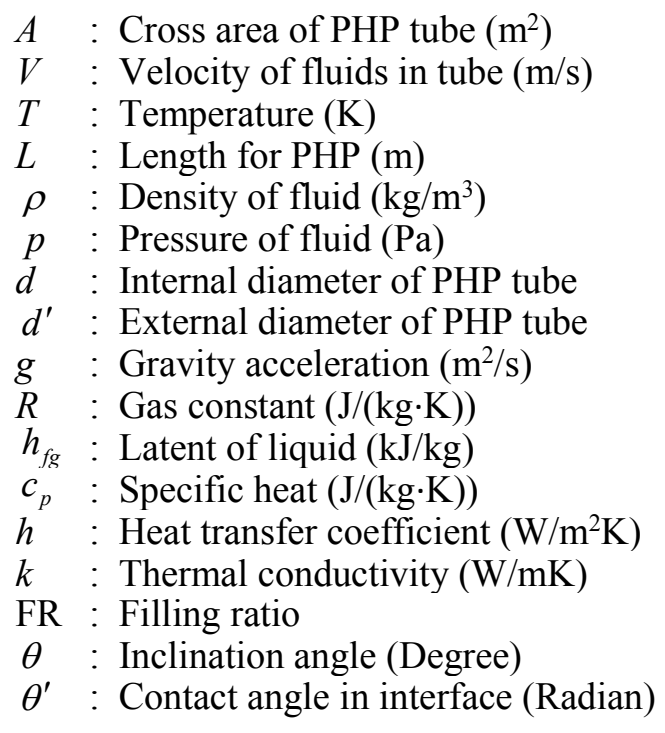

\section{REFERENCES}

[1] Xue Z. H., Xie M. H., Qu W., et al., Ammonia pulsating heat pipe: full visualization and thermal performance, in: Proceeding of the $11^{\text {th }}$ International Heat Pipe Symposium (11IHPS), Beijing, China, 2013, pp. 98-103.

[2] Xue Z. H., Qu W., Xie M. H., Full visualization and startup performance of an ammonia pulsating heat pipe, Propulsion and Power Research, 2013, 2(4): 263-268.

[3] Rittidech S., Pipatpaiboon N., Terdtoon P., Heattransfer characteristics of a closed-loop oscillating heat-pipe with check valves, Applied Energy, 2007, 84(5): 565-577.

[4] Chen P., Lee Y., Chang T., Predicting thermal instability in a closed loop pulsating heat pipe 
system, Applied Thermal Engineering, 2009, 29(89): 1566-1576.

[5] Jia L., Li Y., Zhang T.T., et al., Experimental research on a pulsating heat pipe, Journal of Engineering Thermophysics, 2009, 30(11): 19011903.

[6] Lin L., Ponnappan R., Leland J., Experimental investigation of oscillating heat pipes, AIAA Journal of Thermophysics and Heat Transfer, 2011, 15(4): $395-400$.

[7] Nakagawa M., Morikawa E., Koyama Y., et al., Development of thermal control for phased Array Antenna, In: 21st International Communications Satellite System Conference and Exhibit, Washington, USA, April 2003, AIAA 2003-2226.

[8] Gu J., Kawaji M., Futamata R., Microgravity performance of micro pulsating heat pipe, Microgravity Sci. Technol., 2005, 16(2005): 181185.

[9] Zhang Y. W., Faghri A., Advances and unsolved issues in pulsating heat pipes, Heat Transfer Engineering, 2008, 29(1): 20-44.

[10] Iwata N., Ogawa H., Miyazaki Y., Experimental study of temperature controllable oscillating heat pipe in space thermal environment, In: 40th International Conference on Environment Systems, Barcelona, Spain, July 11-15, 2010, AIAA 20106058.

[11] Iwata I., Ogawa H., Miyazaki Y., Temperaturecontrollable oscillating heat pipe, Journal of Thermophysics and Heat Transfer, 2011, 22(3): 386392.

[12] Okazaki S., Fuke H., Ogawa H., et al., Development of a meter-scale U-shaped oscillating heat pipe for GAPS, In: 42nd International Conference on Environmental System, San Diego, California, July 15-19, 2012, AIAA 2012-3499.

[13] Shafii M. , Faghri A. , Zhang Y. W., Thermal modeling of unlooped and looped pulsating heat pipes, ASME Journal of Heat Transfer, 2001, 123(2001): 1159-1172.

[14] Shafii M. B., Faghri A., Zhang Y. W., Analysis of heat transfer in unlooped and looped pulsating heat pipes, Int. J. Num. Meth. Heat Fluid Flow, 2002, 12(5): 589-609.

[15] Zhang Y. W., Faghri A., and Shafii M. B., Analysis of liquid-vapor pulsating flow in a U-shaped miniature tube, Int. J. Heat Mass Transfer, 2002, 45(2002): 2501-2508.

[16] Zhang Y. W., and Faghri A., Oscillatory flow in pulsating heat pipes with arbitrary numbers of turns, AIAA Journal of Thermophysics and Heat Transfer, 2003, 17(2003): 340-347.

[17] Groll M., Khandekar S., Pulsating heat pipes: a challenge and still unsolved problem in heat pipe science, In: Proc. of 3rd Int. Conf. on Transport Phenomena in Multi-phase Systems, Poland, 2002.
[18] Ma H. B., Maschmann M. R., and Liang S. B., Heat transport capability in a pulsating heat pipe, AIAA 2002-2765, 2002.

[19] Qu W., Qu Y. T., and Ma T. Z., Mechanisms of coupled heat transfer and flow of high heat flux pulsating heat pipe, In: Proc. of 2nd Int. Conf. on Microchannels and Minichannels, New York, USA, 2004.

[20] Qu W, Ma H. B., Theoretical analysis of startup of a pulsating heat pipe. Int. J. Heat Mass Transfer 2007, 50(2007): 2309-2316.

[21] Gu J., Kawaji M., and Futatmata R., Effect of gravity on the performance of pulsating heat pipe, AIAA Journal of Thermophysics and Heat Transfer, 2004, 18(2004): 370-378.

[22] Mameli M. ,Marengo M., Zinna S., Numerical investigation of the effects of orientation and gravity in a closed loop pulsating heat pipe, Microgravity Sci. Technol., 2012, 24(2012):79-92.

[23] Su Y., Davidson J.H., Kulacki F.A., Numerical investigation of fluid flow and heat transfer of oscillating pipe flows, International Journal of Thermal Sciences, 2012, 54(2012): 199-208.

[24] Gi K, Sato F, Maezawa, S., Flow visualization experiment on oscillating heat pipe. In: Proceedings of the 11th International Heat Pipe Conference, Tokyo, Japan, 1999, pp. 149-153.

[25] Tong B. Y., Wong T. N., Ooi K. T., Closed loop pulsating heat pipe, App. Therm. Eng., 2001, 21(2001): 1845-1862.

[26] Lin L., Ponnappan R., and Leland J., Experimental investigation of oscillating heat pipe, AIAA Journal of Thermophysics and Heat Transfer, 2001, 15(4): 395-400.

[27] Charoensawan P., Khandekar S., Groll M., Closed looped pulsating heat pipes Part A: parametric experimental investigation, App. Therm. Eng., 2003, 23(2003): 2009-2020.

[28] Khandekar S, Charoensawan P, Groll M. Closed looped pulsating heat pipes: part B: visualization and semi-empirical modeling. Applied Thermal Engineering 2003, 23(2003): 2021-2033.

[29] Zhang X. M., Xu J. T., and Zhou Z. Q., Experimental study of a pulsating heat pipe using FC-72, ethanol, and water as working fluids, Experimental Heat Transfer, 2004, 17(1): 47-67.

[30] Xu J. L., Li Y. X., Wong T. N., High speed flow visualization of a closed loop pulsating heat pipe, Int. J. Heat Mass Transfer, 2005, 48(16): 3338-3351.

[31] Qu W., Zhou Y., Li Y., et al., Experimental study on mini pulsating heat pipe with square and regular triangle capillaries, In: Proc. 14th International Heat Pipe Conference, Florianopolis, Brazil, 2007.

[32] Song Y. X., Xu J. L., Chaotic behavior of pulsating heat pipes, International Journal of Heat and Mass Transfer 2009, 52(2009): 2932-2941.

[33] Wilson C., Borgmeyer B., Jacobson D., et al., Thermal and visual observation of water and 
acetone oscillating heat pipes, ASME J. Heat Transfer, 2011, 133(6), 061502: 1-5.

[34] Yoon I. , Wilson C. , Borgmeyer B., Neutron phase volumetry and temperature observations in an oscillating heat pipe, International Journal of Thermal Sciences, 2012, 60(2012): 52-60.

[35] Xue Z. H., Qu W., Experimental study on effect of inclination angles to ammonia pulsating heat pipe, Chinese Journal of Aeronautics, 2014, 27(5): 11221127.

[36] Taft B. S., Williams A. D., Drolen B. L., Review of pulsating heat pipe working fluid selection, AIAA J. Thermophysics and Heat Transfer, 2012, 26(4): 651656.

[37] Chato, J. C., Laminar condensation inside horizontal and inclined tubes, ASHRAE J. 1962, 4(2): 52-60.

[38] Jaster, H., Kosky, P. G., Condensation in a mixed flow regime, Int. J. Heat Mass Transfer 19, pp. 9599, 1976.

* Corresponding author.

Email address: xuezhihu9@hotmail.com
[39] Shah, M. M., A general correlation for heat transfer during film condensation inside pipes, Int. J. Heat Mass Transfer 22, pp. 547-556, 1979.

[40] Dobson, M. K., Chato, J. C., Condensation in smooth horizontal tubes, J. Heat Transfer, ASME 120, pp. 193-213, 1998.

[41] Cavallini, A., Del Col, D., Doretti, L., et al., Condensation in horizontal smooth tubes: a new heat transfer model for heat exchanger design, Heat Transfer Eng., 2006, 27(8): 31-38.

[42] Shah, M. M., A new flow pattern based general correlation for heat transfer for heat transfer during condensation in horizontal tubes, In: Proceeding of the 15th International Heat Transfer Conference, Kyoto, Japan, August 10-15, 2014.

[43] Beithier J., Microdrops and Digital Microfluidics, William Andrew, NY USA, 2008.

[44] Darby, R., Correlate pressure drops through fittings, Chem. Eng., 1999, 106(7): 101-104.

[45] Darby, R., Correlate pressure drops through fittings, Chem. Eng., 2001, 108(4): 127-130. 